\title{
Neutrons and the Crystal Ball Experiments
} EIVEQ

CB-97-009

OCT 131999

\section{USTI}

\author{
J. Alyea,${ }^{1,2}$ D. Grosnick, ${ }^{1}$ D. Koetke,${ }^{1}$ R. Manweiler,${ }^{1,2}$ \\ H. Spinka, ${ }^{2}$ and S. Stanislaus ${ }^{1}$
}

${ }^{1}$ Departinent of Physies and Astronomy, Valparaiso University, Valparaiso, IN 46383

and

${ }^{2}$ High Energy Physics Division, Argonne National Laboratory, Argonne, IL 60439

October 15,1997

\section{Introduction}

The Crystal Ball detector, as originally constructed, consisted of a set of 672 optically-isolated $\mathrm{NaI}$ crystals, forming an approximately spherical shell and each crystal viewed by a photomultiplier, a. charged-particle tracker within the $\mathrm{NaI}$ shell, and two endcaps to cover angles close to two colliding beams. The detector geometry subtends a solid angle of about $93 \%$ of $4 \pi$ si $\left(20^{\circ} \leq \theta \leq 160^{\circ}\right.$ and $\left.0^{\circ} \leq \phi \leq 360^{\circ}\right)$ from the center. The Crystal Ball detector was used for two long series of experiments at the $e^{+} e^{-}$ colliding beam accelerators SPEAR $[1,2,3,4]$ at SLAC and DORIS $[5,6,7,8]$ at DESY.

A new set of measurements using the Crystal Ball detector is planned at the Brookhaven National Laboratory Alternating Gradient Synchrotron (BNL AGS). These new experiments will use the $672 \mathrm{NaI}$ crystals from the original detector, but neither the tracker nor endcaps. The "Crystal Ball" in this note will refer only to the set of $\mathrm{NaI}$ crystals. Initially, the reactions to be studied will include $\pi^{-} p \rightarrow$ neutrals with pion beam momenta $\sim 400-750 \mathrm{MeV} / \mathrm{c}$ and $K^{-} p \rightarrow$ neutrals with kaon beam momenta 


\section{DISCLAIMER}

This report was prepared as an account of work sponsored by an agency of the United States Government. Neither the United States Government nor any agency thereof, nor any of their employees, make any warranty., express or implied, or assumes any legal liability or responsibility for the accuracy, completeness, or usefulness of any information, apparatus, product, or process disclosed, or represents that its use would not infringe privately owned rights. Reference herein to any specific commercial product, process, or service by trade name, trademark, manufacturer, or otherwise does not necessarily constitute or imply its endorsement, recommendation, or favoring by the United States Government or any agency thereof. The views and opinions of authors expressed herein do not necessarily state or reflect those of the United States Government or any agency thereof. 


\section{DISCLAIMER}

Portions of this document may be illegible in electronic image products. Images are produced from the best available original document. 
$\sim 600-750 \mathrm{MeV} / \mathrm{c}$. Each of these reactions will include a neutron in the final state. whereas the fraction of $e^{+} e^{-}$interactions with neutrons at SLAC or DESY was quite small. Consequently, there is relatively little experience understanding the behavior of neutrons in the Crystal Ball.

This note will describe some studies of the effects of neutrons in the Crystal Ball for the planned AGS experiments ${ }^{1}$. In Sec. 2, a summary of past measurements with neutrons in $\mathrm{NaI}$ is given along with a description of the approximate detection efficiencies and the response for neutrons and photons. In Sec. 3, the studies of the kinematics for the $\pi^{-} p$ and $K^{-} p$ interactions are presented, and plots showing the expected neutron energies and the angles at which the neutrons are emitted from the reactions are presented. Results from some computer simulations of the Crystal Ball response to neutrons are then given in Sec. 4. This is followed in Sec. 5 by a description of the plastic scintillation neutron counters that might be used with the Crystal Ball, including sizes, expected resolutions and efficiencies, and the associated electronics that would be needed for these detectors. Possible configurations for these neutron counters are discussed, and a preferred configuration suggested. Finally, a summary and comments on the data analysis for the neutrons are presented.

This note attempts to address several concerns related to neutrons and the Crystal Ball detector. A large fraction of the events to be studied contain neutrons that are emitted at angles subtended by the Crystal Ball. This is due to the large detector acceptance and the presence of a neutron in every event for the $\pi^{-} p \rightarrow$ neutrals and $K^{-} p \rightarrow$ neutrals reactions. As will be shown, the neutron will interact in the Crystal Ball (the $\mathrm{NaI}$ thickness corresponds to $\sim 1$ interaction length) for a substantial fraction of the events, and the pattern of energy deposition per event within the NaI crystals mar. appear consistent with that for a photon. Such interactions will certainly complicate the data analysis, and could conceivably lead to the rejection of many erents from the data sample. This conclusion assumes that GEANT reasonably describes the detector

\footnotetext{
${ }^{1}$ Most of this report was written by December 1996, before the experimental runs. Various delays have occurred in finalizing the report, so that the runs are now past. However, the text was not changed to take this into account, only to correct some factual errors.
} 
response.

There will also be background events detected by the Crystal Ball from many other sources. These backgrounds are not possible to simulate, since the particle types, energy spectrum. directions, time distributions, etc. are all unknown. Thus, it may be prudent to consider the addition of other counters, especially in the forward direction $\left(\theta \leq 20^{\circ}\right)$, corresponding to the "tunnel" along the beam axis in the Crystal Ball. These additional counters could supply more information on a fraction of events, such as those containing energy deposited in the $\mathrm{NaI}$ from background interactions or from neutrons. The present plan calls for the addition of an endcap of CsI for the 1998 or 1999 runs, but not earlier, so the use of existing plastic scintillation neutron counters was considered for experiments in 1997. Such neutron counters have a poor efficiency for the detection of photons and have good timing resolution. Therefore, the time of flight can be used to distinguish between neutrons and photons, while charged-particle veto counters can eliminate protons, $\pi^{ \pm}$. etc. No comparison is made between the neutron and CsI counters for the forward detector; this may be possible after the 1997 runs are analyzed. Rather, the neutron counters are considered to add more information for a fraction of the events in an attempt to better understand the sources of background interactions in the detected and triggered events.

\section{Neutrons in NaI Crystals}

It was expected that the response of $\mathrm{NaI}$ counters to neutrons with kinetic energies less than about $15 \mathrm{MeV}$ would have been well studied in the past. Such neutrons were produced by van de Graaff accelerators for the past several decades, and NaI detectors were frequently used for gamma-ray measurements at these facilities. Thus, it was important to understand the neutron-induced backgrounds for such nuclear-structure studies. However, very little information has been found in the literature for the response or efficiency of $\mathrm{NaI}$ detectors to these low-energy neutrons. At higher neutron energies, other types of detectors have been more common, and consequently there are few references to higher-energy neutrons detected in NaI counters as well. This section describes 
some of the known information and the applications to the Crystal Ball for its use in the upcoming AGS experiments.

The short distance from the experimental target, located at the center of the Crystal Ball, to the $\mathrm{NaI}$ crystals, combined with the intrinsic timing resolution of these counters, does not permit neutrons and photons to be distinguished on the basis of timing, except for energies of about $10 \mathrm{MeV}$ or less. For example, the differences in flight times from the target to the front face of a $\mathrm{NaI}$ crystal (a distance of about 10 in $=25.4 \mathrm{~cm}$ ) between neutrons and photons are $5.0,2.6,1.1$, and $0.45 \mathrm{~ns}$ for the kinetic energies, $T=10,30,100$, and $300 \mathrm{MeV}$, respectively. The corresponding time differences to the rear face of a $\mathrm{Nal}$ cystal (a distance about $26 \mathrm{in}=66.0 \mathrm{~cm}$ ) between neutrons and photons are $13.0,6.7,2.9$, and $1.2 \mathrm{~ns}$. Thus, except at the lowest neutron energies. the time differences are all comparable to the time resolution of the $\mathrm{NaI}$ counters, $\sigma_{t} \geq \mathrm{I}$ ns. In addition, the uncertainty in the depth within the $\mathrm{NaI}$ crystal at which the neutron or photon interacts will make it even more difficult to distinguish the two. For the same energies as above, the time differences between a neutron interacting at the front face of the $\mathrm{NaI}$ and a photon interacting at the back face, corrected for the time for the scintillation light to reach the photomultiplier, are approximately $6.2,3 . \bar{\imath}, 2.3$, and $1.6 \mathrm{~ns}$.

At low energies, up to $60 \mathrm{MeV}$, another technique to distinguish neutrons and photons is pulse-shape discrimination. The decay time of the scintillation light is observed to be measurably different for the two, but this difference only seems to occur for organic scintillators [9]. Differences in pulse shape for NaI, if they exist, appear to be quite small. (Such a statement has not been found in the literature. However, even early instrumentation articles describing pulse-shape differences for organic scintillators. such as Ref. [10], never discuss NaI. Another early reference [11] discusses pulse-shape discrimination for organic scintillators, and the discrimination of gamma-ray interactions from noise pulses in NaI!) It should be noted that there are differences in the physical processes for the production of scintillation light in $\mathrm{NaI}$ and in organic materials [12]. Finally, $\mathrm{NaI}$ counters have also been used for the detection of charged particles [13, 14].

There are also very few references in the literature to neutron interactions in $\mathrm{NaI}$ counters at higher energies. One of the main exceptions is an experiment performed 
at LAMPF by P. Dunphy et al. $[15,16]$. An instrument containing NaI counters was launched on the Solar Maximum Mission satellite to study the energy spectrum of solarflare gamma rays as a function of time. Unexpectedly, neutrons from these flares were also observed, delayed in arrival time from the gamma rays. The LAMPF experiment was performed to measure the detection efficiency for neutrons of energies $T_{n}=36-709 \mathrm{MeV}$. The results were compared to Monte Carlo simulations of their detector in Ref. [16], and only a fair agreement was observed between the simulation and the data.

In Ref. [15], the efficiencies were measured for two thicknesses, 10 and $30 \mathrm{~cm}$, of $\mathrm{NaI}$ at a threshold of $10 \mathrm{MeV}$ (electron equiralent energy); see Table I. These detection efficiencies are divided by the counter thicknesses, and are plotted in Fig. 1 as a function of neutron kinetic energy. It can be seen that above $\sim 100 \mathrm{MeV}$, the efficiency is reasonably flat with a value $\geq 1.0-1.5 \% / \mathrm{cm}$. Using these values and the $40.5 \mathrm{~cm}$ thickness of the Crystal Ball NaI counters, the expected efficiency is approximately $40-60 \%$. Alternately, the efficiencies can be parameterized in terms of coefficients $\lambda$,

$$
\text { Efficiency }=1-e^{-\lambda x}
$$

where $x$ is the material thickness. The $\lambda$ values are given in Table I for the 10 and 30 $\mathrm{cm}$ thicknesses. An average $\lambda$ for each energy is tabulated, and the expected efficiency for a $40.5 \mathrm{~cm}$ thickness is also computed. Again, these expected efficiencies are $\sim 40-60 \%$ for neutron energies above $100 \mathrm{MeV}$. Note that a threshold lower than $10 \mathrm{MeV}$ would give a slightly higher efficiency, especially for lower neutron energies. Also, an energy $T$ deposited by the neutron in the NaI may produce a signal corresponding to an electron equivalent energy smaller than $T$ due to light saturation effects.

While photons will normally deposit all their energy in a localized volume within the Crystal Ball and have a detection efficiency close to $100 \%$, except for energies near the threshold, neutrons will have a wide range of interactions. Some of these neutron interactions will be localized. An example of this is a quasielastic scattering of a neutron from a proton bound in a $\mathrm{Na}$ or I nucleus, where the outgoing proton stops in the Crystal Ball and the neutron escapes without further interaction. In this case, the energy deposition will be localized within the Crystal Ball detector. Alternately, if the scattered neutron 
has sufficient energy and interacts again far from the first scattering, the neutron's energy deposition will be highly nonlocalized. In any case, the detected energy will be less than or equal to the initial neutron kinetic energy, depending on whether the scattered neutron or secondary particles escape from the Crystal Ball. Furthermore, because some of the neutron interactions will be nonlocalized, it will be difficult to know which $\mathrm{NaI}$ counters to include in an energy sum. On the other hand, the time-of-flight method can be used to identify secondary neutron interactions far from the primary scattering.

In conclusion, the energy of the neutron cannot be reliably measured in the Crystal Ball, though its angle of trajectory can be obtained. It generally will not be possible to distinguish neutrons from photons at energies above $10-20 \mathrm{MeV}$ on the basis of time of flight from the target to the Crystal Ball due to time differences that are too small. However, secondary neutron interactions occurring far from the initial scattering can probably be distinguished by time of flight. The efficiency for neutron detection is expected to be about $40-60 \%$ at energies above $100 \mathrm{MeV}$, decreasing in efficiency as the neutron energy approaches the threshold energy used in the experiment. Therefore, the energy deposited in the Crystal Ball by the neutrons may cause significant problems in identifying the original reaction, leading to larger than anticipated uncertainties in the measured differential cross sections.

\section{Kinematics Studies}

In order to evaluate the potential for neutron backgrounds in the upcoming Crystal Ball experiments, simulations of $\pi^{-} p$ and $K^{-} p$ interactions were performed. Neutralparticle decays were taken into account until only photons and neutrons remained, and then the angles and energies of the neutrons were recorded. A few cross checks with analytical calculations were performed, primarily for the simpler two-body reactions.

Events were generated using relativistic kinematics with pion beam momenta of 400,600 , and $750 \mathrm{MeV} / \mathrm{c}$ and with kaon momenta of 600 and $740 \mathrm{MeV} / \mathrm{c}$, to bracket the anticipated range for the AGS experiments. The program DEC_KIN was used to study the kinematics of particle reactions or reaction chains. It can include as input, the 
beam width and target dimensions, as well as the angular distributions of 2- and 3-body final states, and generates the laboratory and C.MI. energies and direction cosines of each particle. Further details on DEC_KIN, including how to install and use, can be found in Ref. [17]. For this study, DEC_KIN was installed and run on UNIX-based SUN Sparcstations. For three or more particles in the final state, events were generated that are uniform in phase space. Decays of neutral strange particles $\left(K_{S}^{0} \rightarrow \pi^{0} \pi^{0}, \Lambda^{0} \rightarrow n \pi^{0}\right.$, and $\left.\Sigma^{0} \rightarrow \Lambda^{0} \gamma\right)$ and of $\pi^{0} \rightarrow \gamma \gamma, \eta^{0} \rightarrow \gamma \gamma$, and $\eta^{0} \rightarrow \pi^{0} \pi^{0} \pi^{0}$ were assumed to be isotropic.

Angular distributions that were measured in previous experiments vere included for the following two-body final states: $\left.\pi^{-} p \rightarrow n \pi^{0}[18,19], \pi^{-} p \rightarrow n \eta^{0}: 20\right], K^{-} p \rightarrow$ $n \overline{K^{0}}[21], K^{-} p \rightarrow \Lambda^{0} \pi^{0}[21]$, and $K^{-} p \rightarrow \Sigma^{0} \pi^{0}$ [21]. For these reactions, Legendrepolynomial fits to the data were adopted to represent the "smoothed" differential cross sections:

$$
\frac{d \sigma}{d \Omega}=\frac{1}{k_{C M}^{2}} \sum_{l=0}^{N} a_{l} P_{l}\left(\cos \theta_{C M}\right)
$$

where $k_{C . M}^{2}$ is the C.M. momentum for the incoming channel. Table II contains the values of $a_{l}$ and $N$ used for each reaction and energy. Some representative cases are illustrated in Fig. 2.

A nonzero beam momentum spread and beam angular divergence were included in the generation of events. At the time of this report, details of the beam-line design were not finalized and thus realistic values for these beam parameters were unknown. Changes to the beam collimation during early data accumulation may also occur; this could influence the final beam divergence. For the calculations presented here, the approximate beam momentum spread was taken to be $\Delta p / p= \pm 2 \%$ and the beam divergence to be $\Delta \theta= \pm 6.3 \mathrm{mrad}$ in both horizontal and vertical directions. These values are expected to apply to the kaon beam runs, or when kaon and pion data are measured simultaneously. Improved momentum spread and divergence are expected when only pion beam events are collected.

Plots of the laboratory scattering angle as a function of neutron kinetic energy for the three pion reactions, $\pi^{-} p \rightarrow n \gamma, \pi^{-} p \rightarrow n \pi^{0}$, and $\pi^{-} p \rightarrow n \eta^{0}$, at pion beam 
momenta of 400,600 , and $750 \mathrm{MeV} / \mathrm{c}$ are shown in Fig. 3 using only generated events. Fig. 6 contains similar results for the $K^{-} p \rightarrow n K_{S}^{0}$ reaction at 600 and $740 \mathrm{MeV} / \mathrm{c}$. These four reactions contain a nearly unique angle-energy relationship. It is not unique because of the beam momentum spread and angular divergence. The maximum neutron laboratory angle is about $90^{\circ}$ for the charge-exchange reactions, $\pi^{-} p \rightarrow n \pi^{0}$ and $\mathrm{K}^{-} p \rightarrow$ $n \overline{K^{0}}$, but it is $180^{\circ}$ for $\pi^{-} p \rightarrow n \gamma$. The threshold for the $\pi^{-} p \rightarrow n \eta^{0}$ reaction is $687 \mathrm{MeV} / \mathrm{c}$, so the kinematic plots are shown only at $750 \mathrm{MeV} / \mathrm{c}$; the maximum neutron angle for that reaction is $21.3^{\circ}$. Finally, the neutron kinematics for $\pi^{-} p \rightarrow n \pi^{0}$ and $\pi^{-} p \rightarrow n \gamma$ are quite similar, as seen in Fig. 3 .

Similar dot plots for the generated events corresponding to the three pion reactions,

$$
\begin{aligned}
& \pi^{-} p \rightarrow n \pi^{0} \pi^{0} \\
& \pi^{-} p \rightarrow n \pi^{0} \pi^{0} \pi^{0} \\
& \pi^{-} p \rightarrow n \pi^{0} \pi^{0} \pi^{0} \pi^{0}
\end{aligned}
$$

are presented in Fig. 4; plots for the four kaon reactions,

$$
\begin{aligned}
K^{-} p & \rightarrow \Lambda^{0} \pi^{0} \\
K^{-} p & \rightarrow \Lambda^{0} \gamma \\
K^{-} p & \rightarrow \Lambda^{0} \eta^{0} \\
K^{-} p & \rightarrow \Lambda^{0} \pi^{0} \pi^{0}
\end{aligned}
$$

are shown in Fig. 5; and plots for the three kaon reactions,

$$
\begin{aligned}
K^{-} p & \rightarrow \Sigma^{0} \pi^{0} \\
K^{-} p & \rightarrow \Sigma^{0} \gamma \\
K^{-} p & \rightarrow n \overline{K^{0}} \pi^{0}
\end{aligned}
$$

are given in Fig. 6. In all of these cases, there is a spread of neutron energies at a particular laboratory angle. The five kaon reactions, $K^{-} p \rightarrow \Lambda^{0} \pi^{0}, \Lambda^{0} \gamma, \Lambda^{0} \pi^{0} \pi^{0}, \Sigma^{0} \pi^{0}$, and $\Sigma^{0} \gamma$, have neutron angles extending to $180^{\circ}$, while all other reactions in Figs. $4-5$ 
have a maximum angle that increases with beam momentum. However, neutrons emitted in the backward hemisphere are all of low energy.

Total cross sections measured in a bubble chamber experiment [21] were used to approximately scale the number of generated events for six of the eight kaon beam reactions. The $K^{-} p \rightarrow \Lambda^{0} \gamma$ and $\Sigma^{0} \gamma$ reactions were excluded because their cross sections are expected to be approximately two orders of magnitude smaller, since they occur via electromagnetic rather than strong interactions. The results from the dot plots of the six reactions were then summed. Nearly all the higher-energy neutrons emerge at angles less than approximately $80^{\circ}$, with the highest energies at the smallest angles. However, there are some lower-encrgy neutrons $\left(T_{n} \leq 15 \mathrm{MeV}\right)$ that have scattering angles greater than $120^{\circ}$. Thus all counters in the Crystal Ball will observe neutrons, though the rate will be higher for the more forward counters.

The dot plot for the six reactions can be summed over the neutron energies to give the relative number of neutrons as a function of angle; see Figs. 7 and S. Three angular ranges are of interest: (1) angles less than $\theta=20^{\circ}$, which contain no Crystal Ball counters, (2) angles between $20^{\circ}$ and $30^{\circ}$, which correspond to the boundary region that is usually not used for the detection of photons since there may be a loss of energy out of the sides of the detector at $\theta=20^{\circ}$, and (3) angles between $30^{\circ}$ and $150^{\circ}$. The backward-going particles with $\theta>150^{\circ}$ are much less numerous than at more forward angles. The boundary regions $\left(20^{\circ}-30^{\circ}, 150^{\circ}-160^{\circ}\right)$ are used to collect energy for photons that strike at $\theta \geq 30^{\circ}$ or $\theta \leq 150^{\circ}$. Figs. 7 and 8 illustrate the number of neutrons in these three angular regions. Approximately $30 \%$ of all neutrons at the $740 \mathrm{MeV} / \mathrm{c}$ kaon beam momentum will be emitted with angles $\theta \leq 20^{\circ}$, and $15 \%$ between $20^{\circ}$ and $30^{\circ}$. The fraction of neutrons within each angular range for each of the eight kaon reactions and two beam momenta are given in Table III for isotropic C.M. angular distributions, and in Table IV with information from the measured differential cross sections included. It can be seen that the fraction of neutrons with $\theta \leq 20^{\circ}$ increases somewhat in Table IV compared to Table III. This is caused by the forward peaking of the angular distributions, as shown in Fig. 2.

Table V contains similar percentages for $\pi^{-} p$ reactions in the three angular ranges 
assuming isotropic C.M. angular distributions. The number of reactions are not normalized by cross sections in the averages, but it can be seen that there will be significant fractions of the interaclions with neutrons in each angular range, just as for the $K^{-} p$ reactions.

\section{Simulations of Neutron Interactions in the Crystal Ball}

In order to gain a qualitative understanding of the neutron interaction "topologies" in the Crystal Ball, the events generated by the DEC_KIN kinematics program were used as input to a GEANT (version 3.21) simulation of the detector. The output of this simulation provides the energy deposited in each $\mathrm{NaI}$ counter for every event. The counters that contain energy from the neutron interaction could be identified. This fact allowed a comparison of photon and neutron interactions.

During the course of this work, it became apparent that some of the cross sections in GEANT were not very accurate. In addition, angular distributions in the C.M. frame were assumed isotropic for the scattered neutron. Members of the University of Regina group plan to improve these cross sections for use by the Crystal Ball collaboration, after which the simulations presented here should be repeated.

The standard Crystal Ball clustering algorithm was used to analyze the simulated photon and neutron interactions in the $\mathrm{NaI}$ counters. A total of 13 (or 12) counters, centered on the counter with the largest energy, is considered a cluster. Twelve counters are used when the largest energy corresponds to a counter on the corner of a "major triangle." Fig. 9 shows the distribution of the ratio in energies,

$$
r_{1}=\frac{\text { energy in the center counter }}{\text { total energy in the cluster }}
$$

and Fig. 10 gives the ratio in energies,

$$
r_{4}=\frac{\text { sum of energies in the } 4 \text { center counters }}{\text { total energy in the cluster }}
$$

for photon and neutron interactions. It can be seen from these plots that, on the average, the energy is more localized for photons than for neutrons. These energy ratios were 
computed for simulated clusters from the $K^{-} p \rightarrow .1^{0} \pi^{0}$ reaction. Thus, the comparisons are not for the same distribution of energies for neutron and photon clusters. However, the general conclusions are expected to be true for other reactions and the same energy distributions.

Finally, Fig. 11 shows the number of crystals per cluster or per neutron from the $K^{-} p \rightarrow \Lambda^{0} \pi^{0}$ reaction. In this case, the photons and neutrons do not seem to be easily distinguished. These results will lead to problems in the reconstruction of the reactions in the Crystal Ball. At present, it is difficult to quantify these problems given the inaccuracies of the neutron cross sections in GEANT. In fact, this may not be possible until actual data become available from the Crystal Ball in the pion and kaon beam experiments.

\section{Plastic Scintillation Neutron Counters}

From the results of the kinematics studies, it appears that there will be significant fractions of neutrons that will not be detected in the Crystal Ball, since the neutrons will emerge in a more forward direction than the $20^{\circ}$ to the nominal beam direction. These fractions are considerably larger than the $\sim 3 \%$ of the $4 \pi$ sr subtended by the forward "tunnel" of the Crystal Ball. Some of these forward neutrons may be detected if additional counters are installed that cover part of this solid angle.

Although it is planned to add CsI detectors at the forward angles after the 1997 running period, it is possible to install some existing plastic scintillator neutron counters before that time. These counters have about a 10-25\% efficiency for detecting eâch neutron, good timing characteristics that allow a crude neutron-energy measurement and a rejection of photons by time of flight, and relatively poor efficiency for photon detection. At least three sets of such plastic scintillation counters exist within the Crystal Ball collaboration that could be used for the AGS experiments. These sets of neutron counters are summarized in Table VI.

One set of 14 counters of various sizes is from the University of Regina and uses 2 in-diameter photomultipliers $[22,23]$. These counters contain rectangular scin- 
tillator and either one or two photomultipliers per counter. The total volume of plastic is $0.040 \mathrm{~m}^{3}$.

Another set of 8 modules consist of three identical, but independent, scintillator blocks $\left(8.9 \times 25.4 \times 25.4 \mathrm{~cm}^{3}\right)$ mounted together to form a volume $26.7 \times 25.4 \times 25.4 \mathrm{~cm}^{3}$. Each block is viewed by two 2 in-diameter photomultipliers from one face. This arrangement allows the rejection of phototube noise, while allowing flexibility in locating the counters to view the target. The total volume of plastic scintillator is $0.138 \mathrm{~m}^{3}$ and the timing resolution was measured to be about $0.5 \mathrm{~ns}$. These counters are from the University of California at Los Angeles (UCLA), and are described further in Refs. [24. 25].

The third set of 26 counters consist of two identical scintillator blocks glued together to give a volume of $11.4 \times 25.4 \times 61.0 \mathrm{~cm}^{3}$. Two 5 in-diameter photomultipliers view the plastic at opposite ends along the long counter dimension. These counters have a total volume of $0.459 \mathrm{~m}^{3}$ and a timing resolution of about $1 \mathrm{~ns}$. They are from Argonne National Laboratory and are described more in Ref. [26].

Measurements of the efficiency of the UCLA and Argonne counters reported in Refs. $[24,25,26]$ give approximate values of $0.7 \% / \mathrm{cm}$ thickness along the neutron direction. Thus, the $25.4 \mathrm{~cm}$ depth of both sets of counters corresponds to a neutron detection efficiency of about $18 \%$, which is somewhat dependent on the energy threshold for the counters. An increased depth, and corresponding increase in efficiency, can be obtained by stacking two or more counters along the neutron direction.

From the kinematics calculations, the highest-energy neutrons for the AGS experiments will be $E_{n} \approx 1400 \mathrm{MeV}\left(T_{n} \approx 460 \mathrm{MeV}\right)$ and $\beta_{n} \approx 0.7424$ from the $\pi^{-} p$ reactions at a pion-beam momentum of $750 \mathrm{MeV} / \mathrm{c}$. In order for these neutrons to be separated from photons by $3 \sigma$, and using, for example, the Argonne neutron counters ( $3 \mathrm{~ns})$,

$$
\Delta(T O F)=\frac{D / c}{\beta_{n}}-\frac{D / c}{1} \geq 3 \mathrm{~ns},
$$

where the distance $D$ from the target to the counters needs to be at least:

$$
D \geq \frac{(3 \mathrm{~ns})(c=30 \mathrm{~cm} / \mathrm{ns})}{\left(\frac{1}{\beta_{n}=0.7424}-1\right)} \approx 260 \mathrm{~cm} .
$$

This will locate the neutron counters outside the "dry room" for the Crystal Ball. 
For the purposes of describing some possible configurations with the available neutron counters, the distance from the target center to the average depth of the counters along the beam direction will be assumed to be $300 \mathrm{~cm}$. This allows some space on the outside of the dry room before the neutron counters, and permits some of the detectors to be stacked two or more deep along the beam. Examples of the configurations considered are shown in Figs. 12-15 (configurations $A-D$ ). In these cases, the counters remain at least $10 \mathrm{~cm}$ from the nominal beam line, or at an angle of $33 \mathrm{mrad}=1.9^{\circ}$, to avoid the tails of the beam spatial distribution near the counters. The angular resolutions at this distance from the target, are $\pm 5-10 \mathrm{~cm} / 300 \mathrm{~cm}= \pm 1-2^{\circ}$ in $\theta$, and $\pm\left(1-2^{\circ} / \sin \theta\right)$ in $\phi$, or comparable to the Crystal Ball angular resolutions.

Fig. 12 illustrates a configuration where the detectors are located to maximize the solid angle subtended, while maintaining at least a $25 \mathrm{~cm}$ depth of plastic along the beam direction, or a neutron-detection efficiency of approximately $18 \%$. The detectors form a $20 \times 20 \mathrm{~cm}^{2}$ hole centered on the beam line. The front faces of some of the counters may need to be offset to make room for photomultipliers and light guides. Some of the smaller counters are not shown; their placement would depend partly on the mounting of other counters.

Figs. 13 and 14 are configurations with counters primarily in the lower half of the available space, but with increased depth $(25-102 \mathrm{~cm})$ over much of the solid angle. Since the events are expected to be uniform in the azimuthal angle $\phi$, this method gives higher neutron-detection efficiency to a subset of the events with neutron scattering angles less than $20^{\circ}$. Fig. 15 shows an intermediate configuration with some regions of increased depth $(51 \mathrm{~cm})$, and others only $25 \mathrm{~cm}$.

The complexity of mounting the counters is worst for configuration $A$ and best for $C$. Calibration with cosmic-ray muons, by requiring three or more counters in a line to have a pulse height above some threshold, will be slightly better in configurations $A$ and $D$. The cosmic-ray peaks were better defined using vertical lines with the Argonne counters, as described in Ref. [26].

A subset of one of these configurations may be all that can be implemented with available electronics. For each of the 120 photomultipliers in the three sets of counters 
in Table VI, a high-voltage, discriminator, and TDC channel is required, and an ADC channel would be very helpful for calibration. Long delay cables are typically needed as well. At this time, it is unknown how many of these electronics channels, cables. and delays will be available.

Based on the discussions given above, none of these configurations is an ideal choice. The largest number of neutrons detected would be using configuration $A$ in Fig. 12, but mounting the neutron counters would be quite complicated. The fewest number of neutrons detected would be using configuration $C$ in Fig. 14, but in this case there is a region with relatively-high neutron-detection efficiency: Configuration $B$ in Fig. 13 involves complicated mounting and an intermediate number of neutrons detected, while configuration $D$ in Fig. 15 would also detect an intermediate number of neutrons.

Given this situation, it was decided to recommend implementation of configuration $D$. The Argonne neutron counters would be stacked two deep, or an efficiency of approximately $30-35 \%$ for neutrons well above the threshold energy. Excluding a $20 \times 20 \mathrm{~cm}^{2}$ area centered on the beam line, the ANL counters subtend about $26 \%$ of the area corresponding to the $20^{\circ}$ exit tunnel of the Crystal Ball. The addition of the University of Regina and UCLA counters will increase this percentage. This configuration also allows implementation of a single counter depth or the lower counters only, if sufficient electronics channels cannot be implemented for all the detectors. Finally, the mounting of the counters should be relatively simple and the method for calibrating the counters with cosmic rays should give good results. It should be emphasized that none of the configurations considered was obviously much better than the others, so the recommendation of configuration $D$ is not very compelling.

\section{Summary}

The Crystal Ball will detect neutrons from the $\pi^{-} p \rightarrow$ neutrals and the $K^{-} p \rightarrow$ neutrals reactions with substantial efficiency. However, accurate information on the neutron energy will not be available, in general. Nor will it be simple to distinguish 
between neutron and photon clusters, except in a statistical manner. Fortunately, timeof-flight methods will allow identification of clusters from secondary reactions far from the primary scattering, as long as there are no photon clusters nearby. Thus, the neutron interactions may lead to problems in the reconstruction of some reactions. The extent of such problems has not been discussed here.

A considerable fraction of the neutrons produced will have laboratory angles, $\theta \leq 20^{\circ}$, though low-enorgy neutrons from some reactions will occur even at backward angles. It is possible to distinguish neutrons from photons at $\theta \leq 20^{\circ}$ using the plastic scintillation counters on the basis of time of flight and also to obtain angular resolutions comparable to those of the Crystal Ball counters. However, the energy resolution for the higher-energy neutrons will be poor. Several configurations of existing counters were considered, and one is (weakly) recommended. For this configuration, shown in Fig. 15, the neutron-detection efficiency is approximately $30-35 \%$ at energies considerably above threshold, and the fraction of the angular region $\theta \leq 20^{\circ}$ subtended is $\geq 26 \%$.

The addition of these plastic scintillation counters to the Crystal Ball will provide further information for a subset of the events collected, which should considerably improve the estimate of certain backgrounds. For example, in the $\pi^{-} p \rightarrow n \gamma$ reaction, a serious background will be the $\pi^{-} p \rightarrow n \pi^{0}$ process where one photon from the $\pi^{0} \rightarrow \gamma \gamma$ decay is not detected or has a very low energy. For the kinematical conditions where the neutron goes forward $\left(\theta \leq 20^{\circ}\right)$ in the laboratory, the identification of the neutron and the measurement of its angle will permit a much cleaner sample of $\pi^{-} p \rightarrow n \gamma$ events.

It should also be mentioned that the neutron-detection efficiency in both the Crystal Ball NaI counters and the plastic scintillation counters can be found from the experimental data. For events that can be cleanly identified with a certain reaction, such as $\pi^{-} p \rightarrow n \pi^{0}$ or $\pi^{-} p \rightarrow n \eta^{0}$, on the basis of the photon clusters alone, the response to the neutrons can be studied. Although this procedure may have some backgrounds, they are not expected to be very significant. 


\section{Acknowledgements}

We wish to thank Chris Allgower of Argonne for assistance with merging the text and figures into this document, and George Lolos and Zisis Papandreou of the University of Regina for information on GEANT simulations of neutron interactions. This work was supported by the U.S. Department of Energy, Division of Medium Energy Nuclear Physics Grant DE-FG02-88ER4016, and by the U.S. Department of Energy, Division of High Energy Physics Contract W-31-109-ENG-38.

\section{References}

[1] M. Oreglia et al., Phys. Rev. D 25, 2259 (1982).

[2] Y. Chan et al., IEEE Trans. Nucl. Sci. NS-25, 333, (1978).

[3] E. D. Bloom and C. W. Peck, Ann. Rev. Nucl. Sci. 33, 143 (1983).

[4] J. E. Gaiser et al., Phys. Rev. D 34, 711 (1986).

[5] D. Antreasyan et al., Phys. Rev. D 36, 2633 (198i).

[6] D. A. Williams et al., Phys. Rev. D 38, 1365 (1988).

[7] H. Marsiske et al., Phys. Rev. D 41, 3324 (1990).

[8] A. Bizzeti et al., Phys. Lett. 267B, 286 (1991).

[9] See for example M. Moszynski et al., Nucl. Instrum. Methods A317, 262 (1992); ibid, A343, 563 (1994); M. Cronqvist et al., Nucl. Instrum. Methods A317, 273 (1992).

[10] F.D. Brooks, Nucl. Instrum. Methods 4, 151 (1959).

[11] M.L. Roush, M.A. Wilson, and W.F. Hornyak, Nucl. Instrum. Methods 31, 112 (1964). 
[12] K. Michaelian, A. Mendhaca-Rocha, and E. Belmont-Moreno, Nucl. Instrum. Methods A356, 297 (1995).

[13] J.L. Romero, G.A. Needham, F.P. Brady, C.MI. Castaneda, and T.D. Ford, Nucl. Instrum. Methods A301, 241 (1991).

[14] J.H. Ha et al., Nucl. Instrum. Methods A350. 411 (1994).

[15] P. P. Dunphy et al., Expt. Astr. 2, 233 (1992).

[16] P. P. Dunphy et al., Nucl. Instrum. Methods A330, 199 (1993).

[17] M. Clajus, "The Crystal Ball Simulation Package," Crystal Ball Note CB-96-1, 1996, (unpublished).

[18] R. M. Brown et al., Nucl. Phys. B 117, 12 (1976); 137, 542 (E) (1978): F. Bulos et al., Phys. Rev. 187, 1827 (1969).

[19] M. E. Sadler et al., Phys. Rev. D35, 2718 (1987).

[20] F. Bulos et al. Phys. Rev. 187, 1827 (1969); R. M. Brown et al., Nucl. Phys. B 153, 89 (1979); W. B. Richards et al., Phys. Rev. Lett. 16, 1221 (1966).

[21] R. Armenteros et al., Nucl. Phys. B 21, 15 (1970).

[22] Z. Papandreou et al., Nucl. Instrum. Methods A268, 179 (1988).

[23] Z. Papandreou et al., Nucl. Instrum. Methods B34, 454 (1988).

[24] J. A. Wightman et al., Nucl. Instrum. Methods A275, 281 (1989).

[25] R. S. Kessler et al., Nucl. Instrum. Methods A275, 285 (1989).

[26] R. Garnett et al., Nucl. Instrum. Methods A309. 508 (1991). 


\section{Tables}

TABLE I. Values of the measured NaI efficiency for neutrons from the reference, P. Dunphy et al. [15]. for the thicknesses of $10.2 \mathrm{~cm}$ and $30.5 \mathrm{~cm}$ and a threshold of $10 \mathrm{MeV}$ (electron equivalent) arc presented. Also given are derived values of the detection coefficients $\lambda$. and the mean detection coefficient $\bar{\lambda}$. The corresponding neutron detection efficiency in the Crystal Ball $(40.5 \mathrm{~cm})$ is shown.

\begin{tabular}{|c||c|c||c|c||c|c|}
\hline \multicolumn{1}{|c||}{$\begin{array}{c}\text { Neutron } \\
\text { K.E. }\end{array}$} & \multicolumn{2}{c||}{$10.2 \mathrm{~cm}$} & \multicolumn{2}{c||}{$30.5 \mathrm{~cm}$} & $\bar{\lambda}$ & $\begin{array}{c}\text { Crystal Ball } \\
\text { Detection }\end{array}$ \\
\cline { 2 - 6 }$(\mathrm{MeV})$ & Efficiency & $\begin{array}{c}\lambda \\
\left(\mathrm{cm}^{-1}\right)\end{array}$ & Efficiency & $\begin{array}{c}\lambda \\
\left(\mathrm{cm}^{-1}\right)\end{array}$ & $\begin{array}{c}\overline{\left(\mathrm{cm}^{-1}\right)} \\
\text { Efficiency }\end{array}$ \\
\hline \hline 36 & 0.048 & 0.0048 & 0.101 & 0.0035 & 0.0041 & 0.153 \\
65 & 0.096 & 0.0099 & 0.233 & 0.0087 & 0.0093 & 0.314 \\
112 & 0.167 & 0.0180 & 0.358 & 0.0145 & 0.0162 & 0.481 \\
207 & 0.175 & 0.0189 & 0.432 & 0.0186 & 0.0187 & 0.531 \\
255 & 0.166 & 0.0179 & 0.447 & 0.0194 & 0.0187 & 0.531 \\
289 & 0.197 & 0.0216 & 0.421 & 0.0179 & 0.0197 & 0.550 \\
365 & 0.180 & 0.0195 & 0.453 & 0.0198 & 0.0197 & 0.550 \\
435 & 0.206 & 0.0227 & 0.487 & 0.0219 & 0.0223 & 0.595 \\
590 & 0.235 & 0.0264 & 0.507 & 0.0232 & 0.0248 & 0.634 \\
709 & 0.232 & 0.0260 & 0.529 & 0.0247 & 0.0253 & 0.641 \\
\hline
\end{tabular}


TABLE II. Values of the parameters, $a_{l}$, of the Legendre polynomial fits to the cross section data. The first contains data for the three reactions, $K^{-}+p \rightarrow n+\overline{K^{0}}$, $K^{-}+p \rightarrow \Lambda+\pi^{0}$, and $K^{-}+p \rightarrow \Sigma^{0}+\pi^{0}$, at the two beam momenta 600 and $740 \mathrm{MeV} / \mathrm{c}$ from Ref. [21]. The second contains data for the $\pi^{-}+p \rightarrow n+\pi^{0}$ reaction at three beam momenta, 400,600, and $750 \mathrm{MeV} / \mathrm{c}[18,19]$, and the $\pi^{-}+p \rightarrow n+\eta$ reaction at a beam momentum of $750 \mathrm{MeV} / \mathrm{c}$ [20].

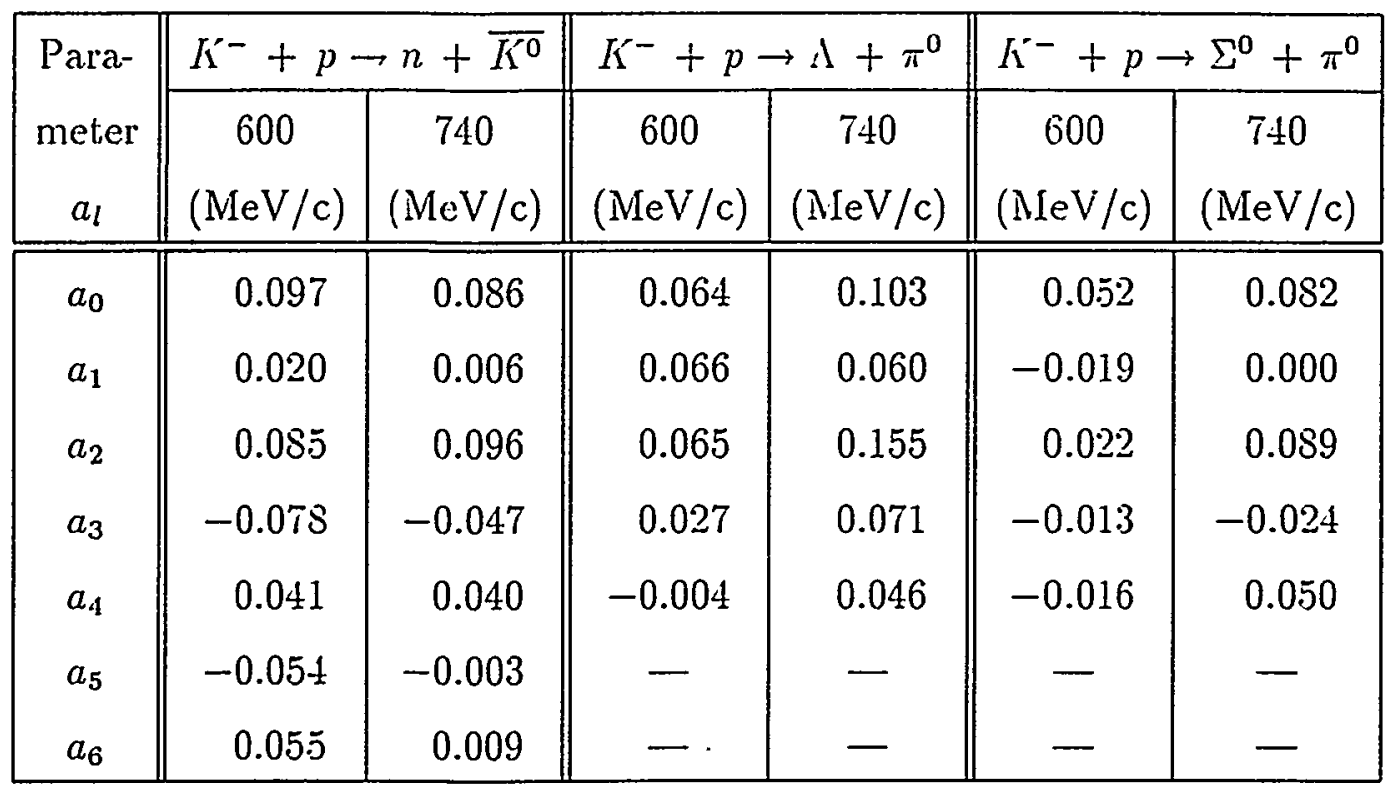

\begin{tabular}{|c||c|c|c||c|}
\hline \multirow{2}{*}{$\begin{array}{c}\text { Para- } \\
\text { meter } \\
a_{l}\end{array}$} & \multicolumn{2}{|c||}{$\pi^{-}+p \rightarrow n+\pi^{0}$} & $\pi^{-}+p \rightarrow n+\eta$ \\
\cline { 2 - 5 } & $\begin{array}{c}400 \\
(\mathrm{MeV} / \mathrm{c})\end{array}$ & $\begin{array}{c}600 \\
(\mathrm{MeV} / \mathrm{c})\end{array}$ & $\begin{array}{c}750 \\
(\mathrm{MeV} / \mathrm{c})\end{array}$ & $\begin{array}{c}750 \\
(\mathrm{MeV} / \mathrm{c})\end{array}$ \\
\hline \hline$a_{0}$ & 0.177 & 0.264 & 0.312 & 0.063 \\
$a_{1}$ & 0.041 & 0.449 & 0.319 & 0.020 \\
$a_{2}$ & 0.122 & 0.353 & 0.417 & 0.025 \\
$a_{3}$ & -0.056 & 0.166 & 0.145 & - \\
$a_{4}$ & - & 0.083 & 0.026 & - \\
$a_{5}$ & - & 0.034 & 0.136 & - \\
$a_{6}$ & - & 0.028 & -0.069 & - \\
\hline
\end{tabular}


TABLE III. List, of $K^{-} p$ reactions at 600 and $740 \mathrm{MeV} / \mathrm{c}$ from Monte Carlo calculations, that do not include differential cross sections. Shown are the percentage of events with neutrons emerging with laboratory angle less than $20^{\circ}$. between $20^{\circ}$ and $30^{\circ}$, and for angles greater than $30^{\circ}$. The total includes only those reactions indicated $(*)$ for which the total cross section is available and not negligible.

\begin{tabular}{|l||c|c|c|r|}
\hline $\begin{array}{l}600 \mathrm{MeV} / \mathrm{c} \\
\text { Reaction }\end{array}$ & $\leq 20^{\circ}$ & $20^{\circ}<\theta \leq 30^{\circ}$ & $>30^{\circ}$ & No. Events \\
\hline \hline$\Lambda \gamma$ & - & - & - & - \\
$\Lambda \pi^{0} *$ & 14.20 & 15.09 & 70.71 & $2 \tau, 000$ \\
$\Lambda \eta(2 \gamma)$ & - & - & - & - \\
$\Lambda \eta\left(3 \pi^{0}\right)$ & - & - & - & - \\
$\Lambda \pi^{0} \pi^{0} *$ & 31.91 & 26.66 & 41.43 & 11,000 \\
$\Sigma \gamma$ & - & - & - & - \\
$\Sigma \pi^{0} *$ & 18.68 & $21.2 \tau$ & 60.05 & 21,000 \\
$n K_{S}^{0} *$ & 13.12 & 14.71 & 72.17 & 40,000 \\
$n K_{S}^{0} \pi^{0} *$ & 78.90 & 21.10 & 0.00 & 1,000 \\
\hline Total * & 17.30 & $17.5 \tau$ & 65.13 & 100,000 \\
\hline
\end{tabular}




\begin{tabular}{|c|c|c|c|c|}
\hline $\begin{array}{l}7+0 \mathrm{MeV} / \mathrm{c} \\
\text { Reaction }\end{array}$ & $\leq 20^{\circ}$ & $20^{\circ}<\theta \leq 30^{\circ}$ & $>30^{\circ}$ & No. Events \\
\hline$\Lambda \gamma$ & 14.79 & 16.16 & 69.05 & 10,000 \\
\hline$\Lambda \pi^{0}$ & $15.0^{\circ} 2$ & 16.57 & 68.40 & 31,000 \\
\hline$\Lambda \eta(2 \gamma) *$ & 87.36 & 12.63 & 0.01 & 7,507 \\
\hline$\Lambda \eta\left(3 \pi^{0}\right)$ & 一 & - . & - & - \\
\hline$\Lambda \pi^{0} \pi^{0} \quad *$ & $33.6: 3$ & 28.15 & 38.22 & 8,750 \\
\hline$\Sigma \gamma$ & 18.14 & 22.00 & 59.86 & 10,000 \\
\hline$\Sigma \pi^{0}$ & 19.28 & 22.92 & 57.79 & 24,700 \\
\hline$n K_{L}^{0}$ & 13.69 & 15.13 & 71.18 & 10,000 \\
\hline$n K_{S}^{0}$ & 14.01 & 14.71 & 71.29 & 25,900 \\
\hline$n K_{L}^{0} \pi^{0}$ & 48.75 & 34.30 & 16.95 & 10,000 \\
\hline$n K_{S}^{0} \pi^{0} \quad *$ & 51.23 & 32.92 & 15.85 & 1,300 \\
\hline Total & 23.41 & 18.60 & 57.98 & 99,157 \\
\hline
\end{tabular}


TABLE IV. List of $K^{-} p$ reactions at 600 and $740 \mathrm{MeV} / \mathrm{c}$ from Monte Carlo calculations, including the differential cross sections $(\diamond)$ for the reaction if available. Shown are the percentage of events with neutrons emerging with laboratory angle less than $20^{\circ}$, between $20^{\circ}$ and $30^{\circ}$, and for angles greater than $30^{\circ}$.

\begin{tabular}{|c|c|c|c|c|}
\hline $\begin{array}{l}600 \mathrm{MeV} / \mathrm{c} \\
\text { Reaction }\end{array}$ & $\leq 20^{\circ}$ & $20^{\circ}<\theta \leq 30^{\circ}$ & $>30^{\circ}$ & No. Events \\
\hline$\Lambda$ & - & - & - & - \\
\hline$\Lambda \pi^{0}$ & 8.50 & 9.25 & 82.25 & 34.600 \\
\hline$\Lambda \eta(2 \gamma)$ & - & - & - & - \\
\hline$\Lambda \pi^{0} \pi^{0} \quad \diamond$ & 31.32 & 27.35 & 41.34 & 14,200 \\
\hline$\Sigma \gamma$ & - & - & - & - \\
\hline$\Sigma \pi^{0}$ & 26.94 & 22.23 & 50.83 & 27,850 \\
\hline$n H_{S}^{\circ 0}$ & 21.45 & 7.11 & 71.44 & 23.000 \\
\hline$n K_{S}^{0} \pi^{0} \diamond$ & 81.71 & 18.29 & 0.00 & 350 \\
\hline Total & 20.11 & 14.97 & 64.92 & 100,000 \\
\hline
\end{tabular}

\begin{tabular}{|c|c|c|c|c|c|}
\hline $\begin{array}{l}740 \mathrm{MeV} / \mathrm{c} \\
\text { Reaction }\end{array}$ & & $\leq 20^{\circ}$ & $20^{\circ}<\theta \leq 30^{\circ}$ & $>30^{\circ}$ & No. Events \\
\hline$\Delta \gamma$ & & - & - & - & - \\
\hline$\Lambda \pi^{0}$ & $\diamond$ & 18.22 & 13.07 & 68.71 & 36,600 \\
\hline$\Lambda \eta(2 \gamma)$ & $\diamond$ & 86.99 & 13.01 & 0.00 & 4,949 \\
\hline$\Lambda \eta\left(3 \pi^{0}\right)$ & $\circ$ & 88.17 & 11.83 & 0.00 & 3,871 \\
\hline$\Lambda \pi^{0} \pi^{0}$ & $\diamond$ & 33.09 & 27.52 & 39.39 & 10,100 \\
\hline$\Sigma_{\gamma}$ & & - & - & - & - \\
\hline$\Sigma \pi^{0}$ & $\diamond$ & 29.08 & 17.68 & 53.24 & 29,260 \\
\hline$n K_{S}^{0}$ & $\diamond$ & 25.57 & 9.36 & 65.07 & 13,400 \\
\hline$n K_{S}^{0} \pi^{0}$ & $\diamond$ & 51.09 & 30.94 & 17.97 & 640 \\
\hline Total & $\diamond$ & 30.35 & 15.47 & 54.18 & 98,820 \\
\hline
\end{tabular}


TABLE V. List of $\pi^{-} p$ reactions at 400,600 , and $i 50 \mathrm{MeV} / \mathrm{c}$ from Monte Carlo calculations. Shown are the percentage of events with neutrons emerging with laboratory angle less than $20^{\circ}$, between $20^{\circ}$ and $30^{\circ}$, and for angles greater than $30^{\circ}$. No differential cross sections have been included and there is no spread in beam momentum.

\begin{tabular}{|l||c|c|c|r|}
\hline $\begin{array}{l}400 \mathrm{MeV} / \mathrm{c} \\
\text { Reaction }\end{array}$ & $\leq 20^{\circ}$ & $20^{\circ}<\theta \leq 30^{\circ}$ & $>30^{\circ}$ & Yo. Events \\
\hline \hline$n \gamma$ & 11.65 & 13.50 & 74.85 & 10,000 \\
$n \pi^{0}$ & 12.45 & 14.18 & 73.37 & 10,000 \\
$n \pi^{0} \pi^{0}$ & 42.98 & 33.22 & 23.80 & 10,000 \\
\hline Total & 22.36 & 20.30 & 57.34 & 30,000 \\
\hline
\end{tabular}

\begin{tabular}{|l||c|c|c|c|}
\hline $\begin{array}{l}600 \mathrm{MeV} / \mathrm{c} \\
\text { Reaction }\end{array}$ & $\leq 20^{\circ}$ & $20^{\circ}<\theta \leq 30^{\circ}$ & $>30^{\circ}$ & No. Events \\
\hline \hline$n \gamma$ & 12.48 & 14.15 & 73.37 & 10,000 \\
$n \pi^{0}$ & 13.55 & 14.55 & 71.90 & 10,000 \\
$n \pi^{0} \pi^{0}$ & 33.27 & 27.84 & 38.89 & 10,000 \\
$n \pi^{0} \pi^{0} \pi^{0}$ & 76.82 & 21.91 & 1.27 & 10,000 \\
\hline Total & 34.03 & 19.61 & 46.36 & 40,000 \\
\hline
\end{tabular}

\begin{tabular}{|l||c|c|c|c|}
\hline \begin{tabular}{l|c}
$750 \mathrm{MeV} / \mathrm{c}$ \\
Reaction
\end{tabular} & $\leq 20^{\circ}$ & $20^{\circ}<\theta \leq 30^{\circ}$ & $>30^{\circ}$ & No. Events \\
\hline \hline$n \gamma$ & 12.98 & 14.81 & 72.21 & 10,000 \\
$n \pi^{0}$ & 14.12 & 15.57 & 70.31 & 10,000 \\
$n \pi^{0} \pi^{0}$ & 30.90 & 25.83 & 43.27 & 10,000 \\
$n \pi^{0} \pi^{0} \pi^{0}$ & 58.64 & 30.55 & 10.81 & 10,000 \\
$n \pi^{0} \pi^{0} \pi^{0} \pi^{0}$ & 99.94 & 0.06 & 0.00 & 10,000 \\
$n \eta$ & 69.69 & 30.31 & 0.00 & 10,000 \\
\hline Total & 47.71 & 19.52 & 32.77 & 60,000 \\
\hline
\end{tabular}


TABLE VI. Dimensions of plastic scintillator neutron counters, number of counters, and number of photomultipliers available for use in the AGS experiments with the Crystal Ball. The first five lines describe counters from the University of Regina. and the sixth and seventh lines describe counters from UCLA and Argonne respectively. The counters with single P.M tubes are oriented with the tubes facing the incident neutron flux. In all cases, the third dimension shown is the thickness which the neutrons would see.

\begin{tabular}{|c|c|c|c|c|}
\hline $\begin{array}{c}\text { Number of } \\
\text { counters }\end{array}$ & $\begin{array}{c}\text { Number of } \\
\text { photomultipliers } \\
\text { per counter }\end{array}$ & $\begin{array}{c}\text { Scintillaior } \\
\text { Dimensions } \\
\left(\mathrm{cm}^{3}\right)\end{array}$ & $\begin{array}{c}\text { Total } \\
\text { Volume } \\
\left(\mathrm{cm}^{3}\right)\end{array}$ & $\begin{array}{c}\text { Total } \\
\text { Numbers of } \\
\text { Photomultipliers }\end{array}$ \\
\hline \hline 4 & 1 & $10 \times 10 \times 30$ & 12,000 & 4 \\
4 & 1 & $10 \times 10 \times 1.5$ & 6,000 & 4 \\
4 & 1 & $10 \times 15 \times 20$ & 12,000 & 4 \\
2 & 2 & $10 \times 20 \times 10$ & 4,000 & 4 \\
2 & 2 & $10 \times 20 \times 15$ & 6,000 & 4 \\
8 & 6 & $26.7 \times 25.4 \times 25.4$ & 138,000 & 48 \\
26 & 2 & $11.4 \times 25.4 \times 61.0$ & 459,000 & 52 \\
& & & $-637,000$ & 120 \\
\hline
\end{tabular}




\section{Figures}

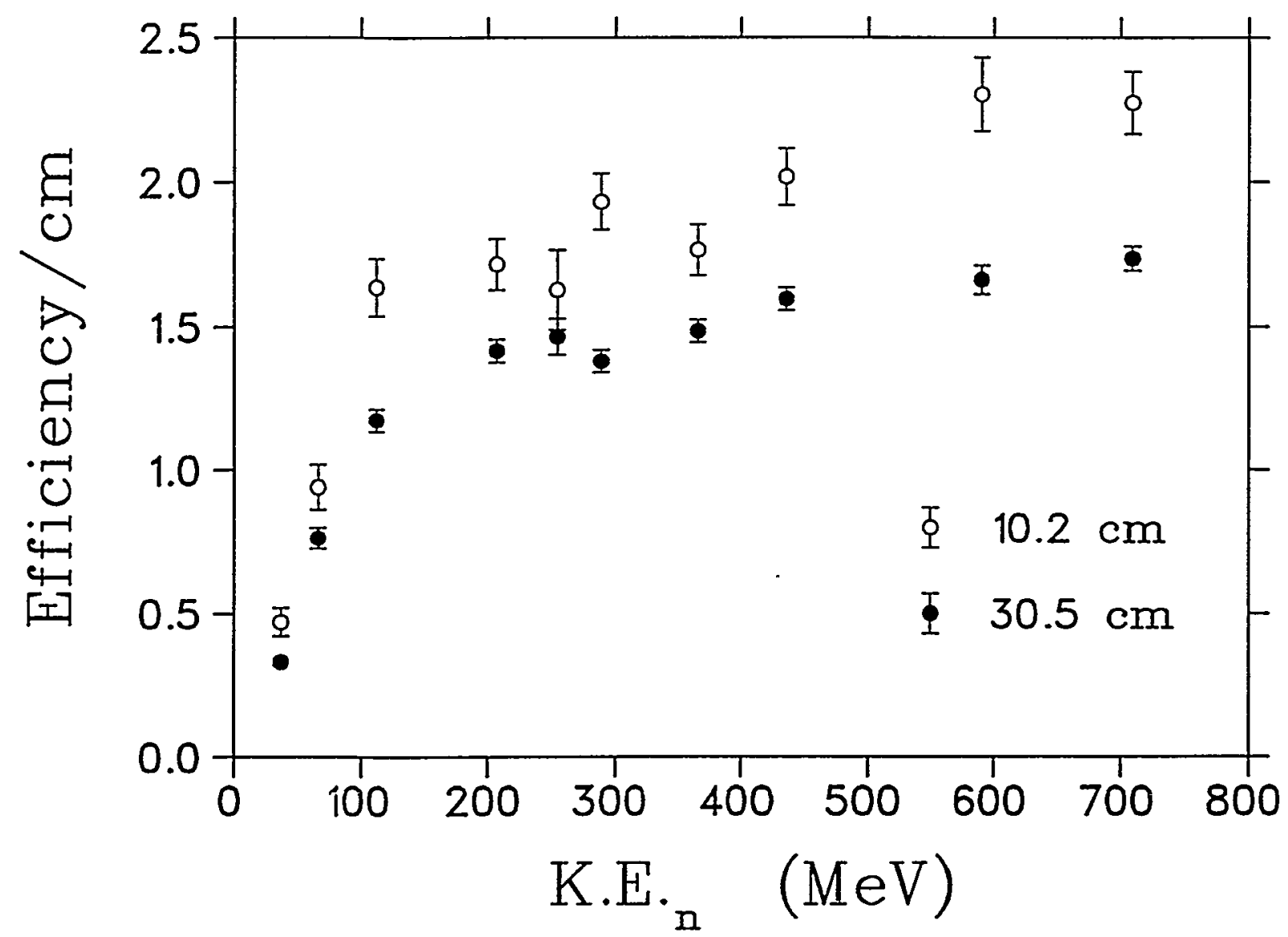

Figure 1: Measured neutron-detection efficiencies per $\mathrm{cm}$ of $\mathrm{NaI}$ as a function of neutron kinetic energy. The results with $10 \mathrm{~cm}$ thickness are given by open circles. and with $30 \mathrm{~cm}$ total thickness by closed circles. The data were obtained from Refs. :15.16], and were collected with a threshold corresponding to $10 \mathrm{MeV}$. 

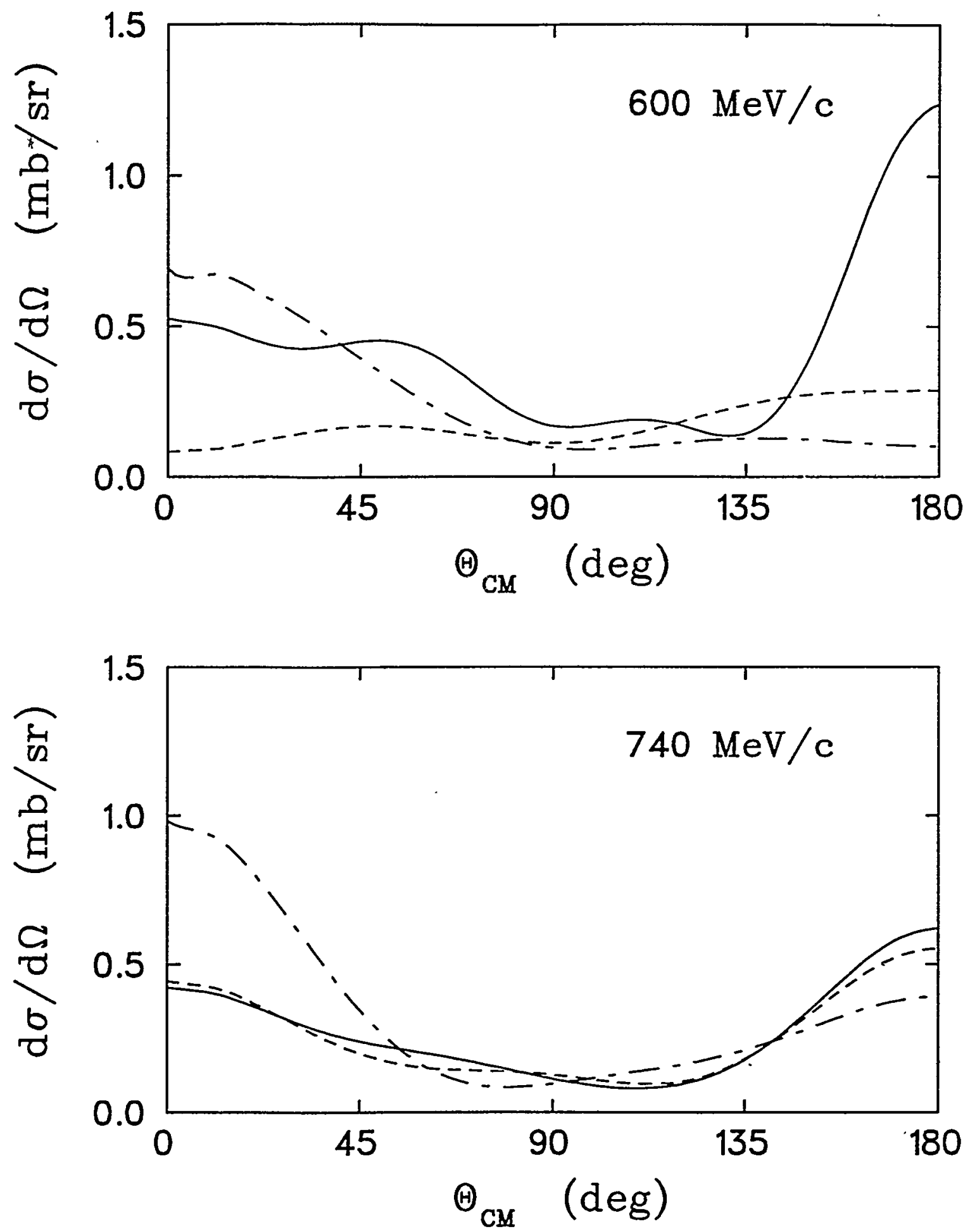

Figure 2: Representative plots of the "smoothed" differential cross section, $d \sigma / d \Omega$, as a function of the C.M. frame neutron scattering angle, $\theta_{C M}$, for $K^{-} p$ reactions at 600 and $740 \mathrm{MeV} / \mathrm{c}$ from Table II and Ref. [21]. The solid line refers to the $K^{-} p \rightarrow n \overline{K^{0}}$ reaction, the dashed line to the $K^{-} p \rightarrow \Sigma^{0} \pi^{0}$ reaction, and the dash-dot line to the $K^{-} p \rightarrow \Lambda^{0} \pi^{0}$ reaction. 

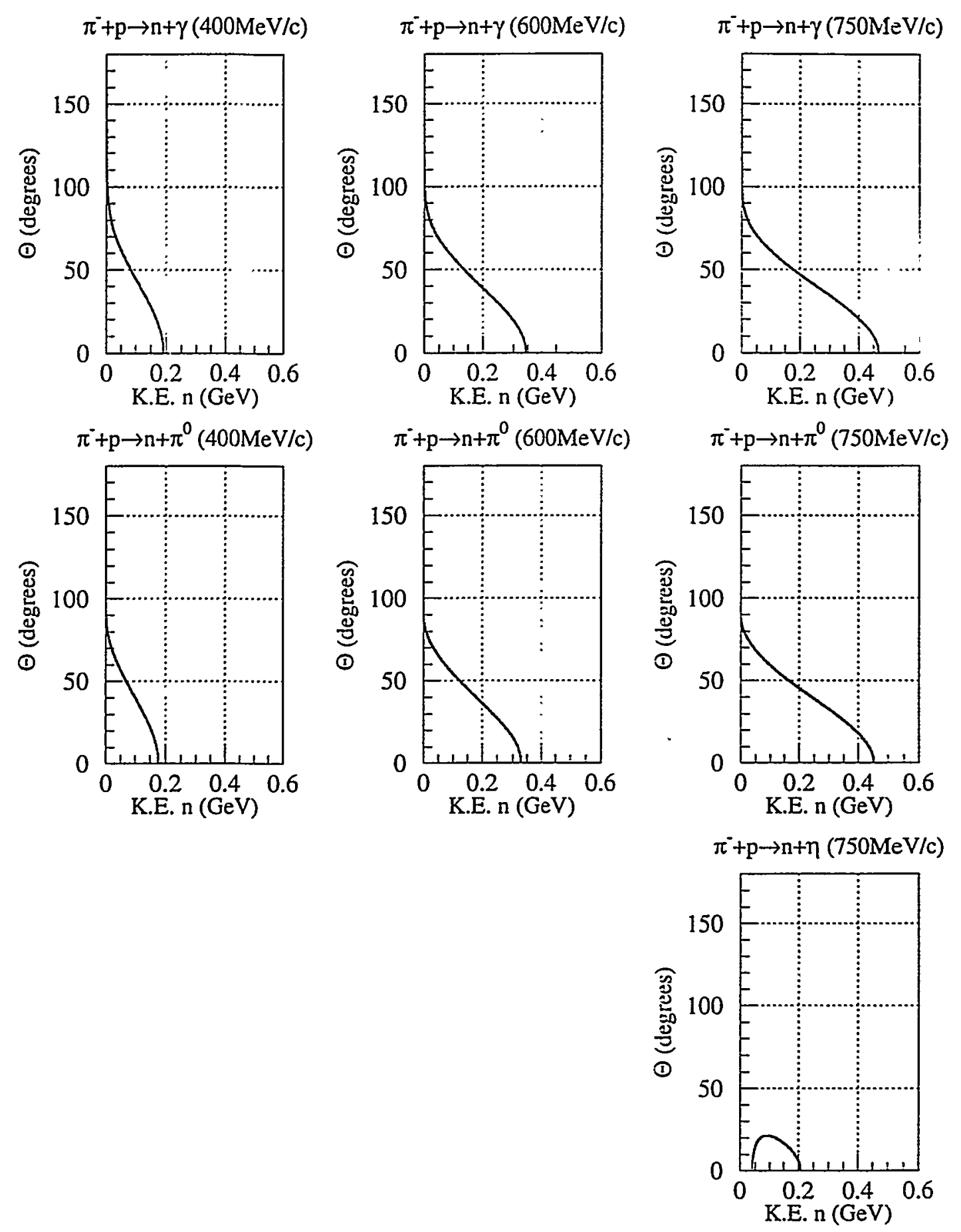

Figure 3: Plots of the neutron laboratory scattering angle $\theta$ versus the laboratory kinetic energy for the three reactions $\pi^{-} p \rightarrow n \gamma, \pi^{-} p \rightarrow n \pi^{0}$, and $\pi^{-} p \rightarrow n \eta^{0}$ at three different beam momenta, 400,600 , and $750 \mathrm{MeV} / \mathrm{c}$. 

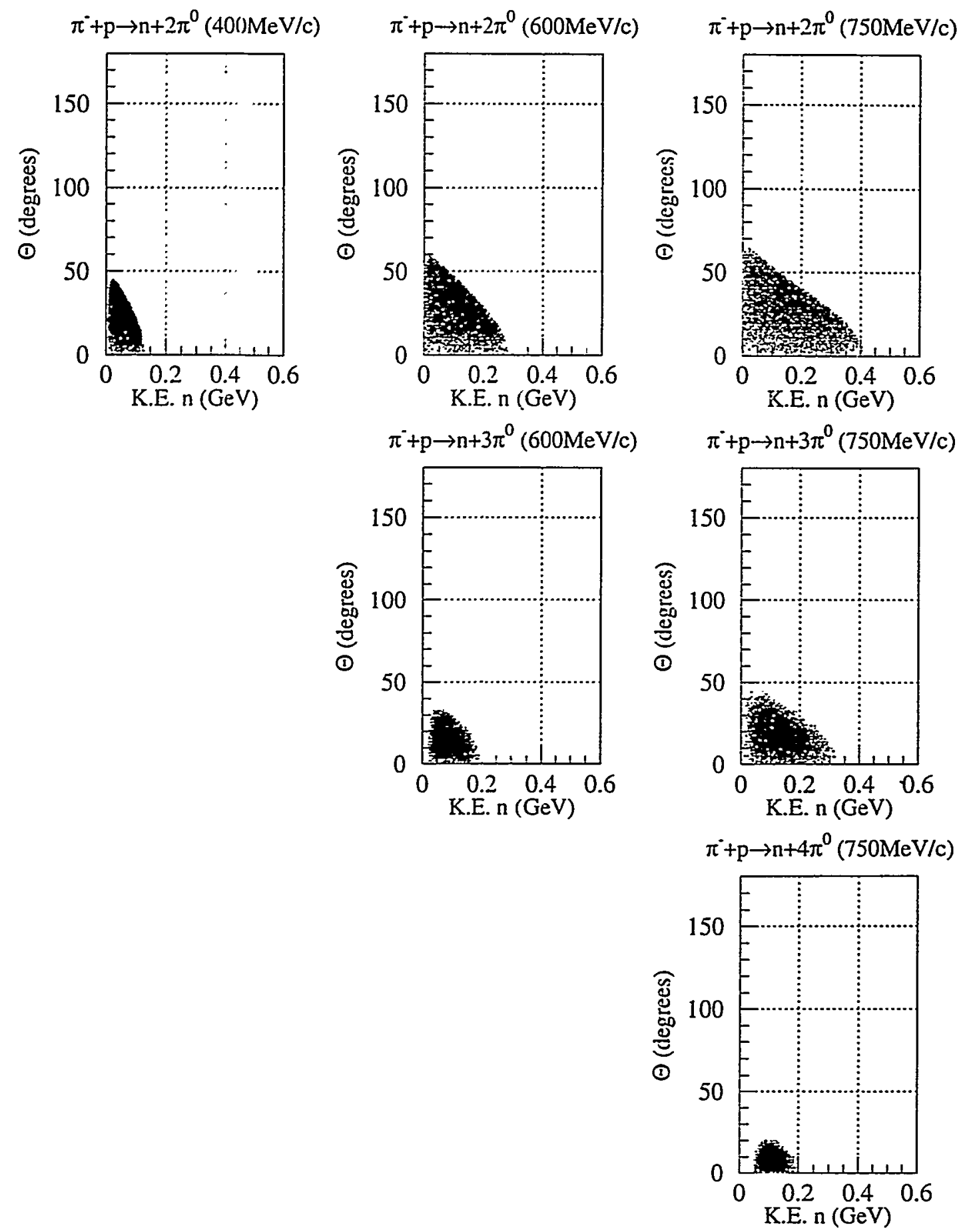

Figure 4: Plots of the neutron laboratory scattering angle $\theta$ versus the laboratory kinetic energy for the three reactions $\pi^{-} p \rightarrow n \pi^{0} \pi^{0} . \pi^{-} p \rightarrow n \pi^{0} \pi^{0} \pi^{0}$, and $\pi^{-} p \rightarrow n \pi^{0} \pi^{0} \pi^{0} \pi^{0}$ at three different beam momenta, 400,600 , and $750 \mathrm{MeV} / \mathrm{c}$. 

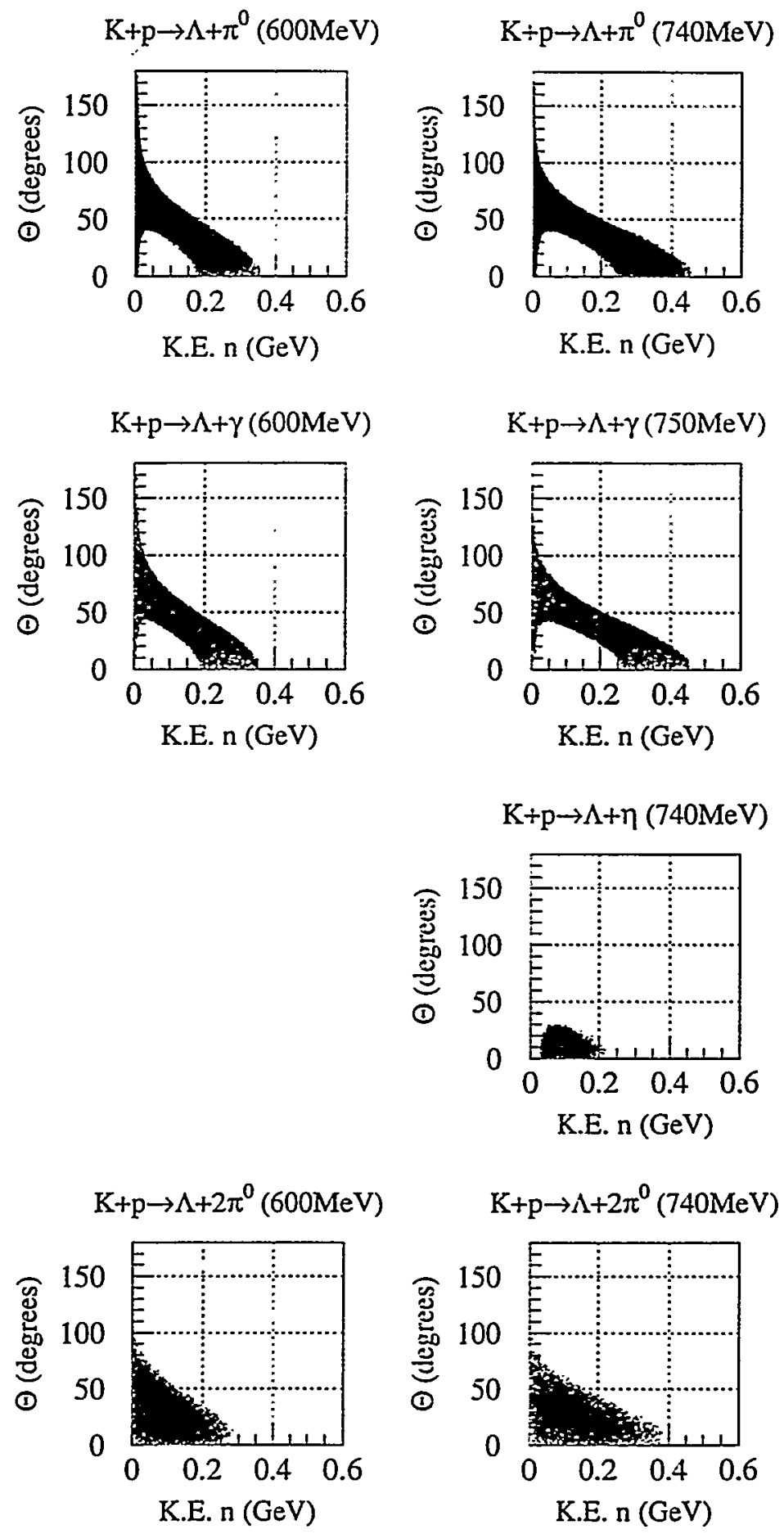

Figure 5: Plots of the neutron scattering angle $\theta$ in the laboratory frame versus the laboratory kinetic energy for the four reactions $K^{-} p \rightarrow \Lambda^{0} \pi^{0}, K^{-} p \rightarrow \Lambda^{0} \gamma, K^{-} p \rightarrow$ $\Lambda^{0} \eta^{0}$, and $K^{-} p \rightarrow \Lambda^{0} \pi^{0} \pi^{0}$ at 600 and $740 \mathrm{MeV} / \mathrm{c}$ incident beam momenta. 

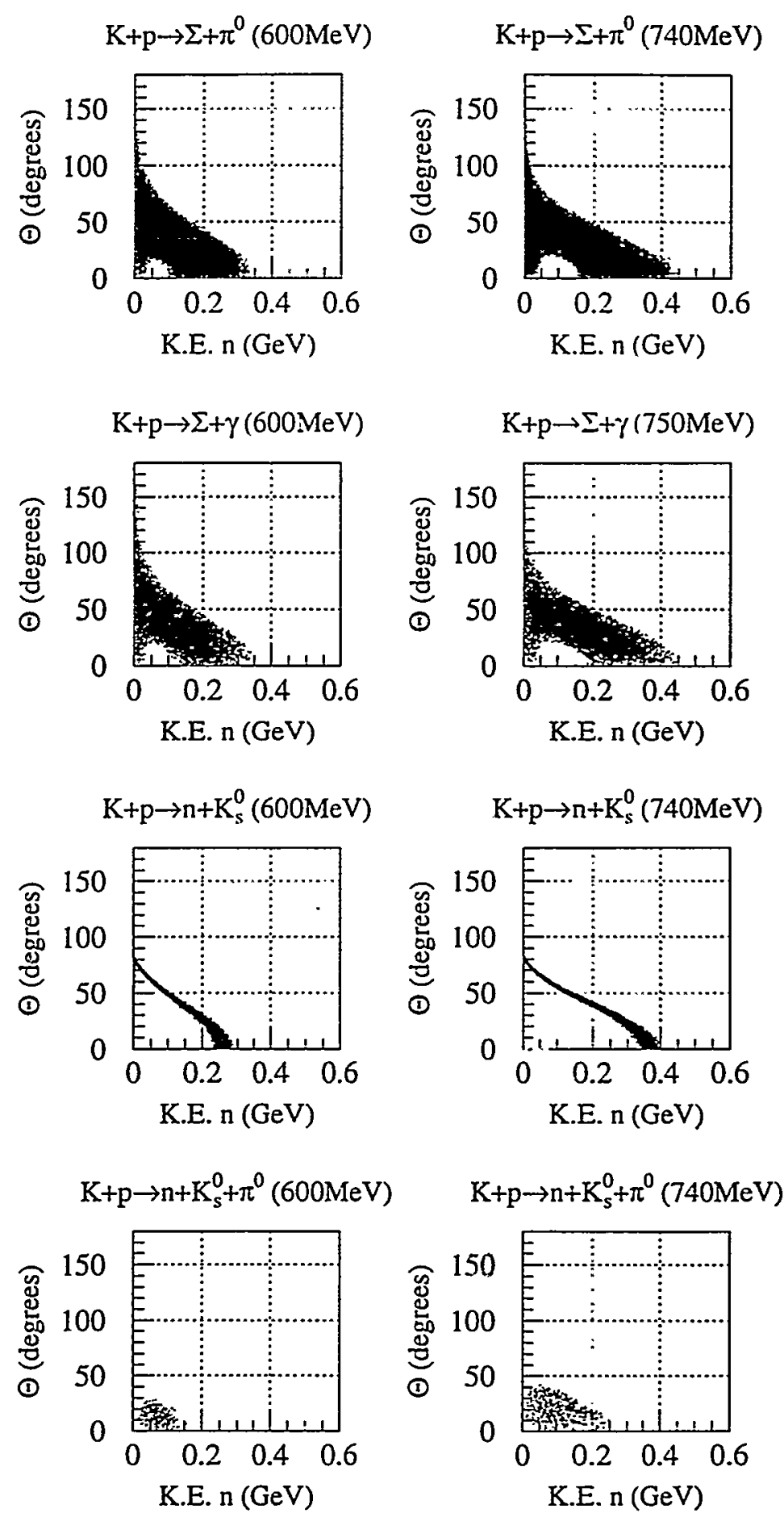

Figure 6: Plots of the neutron scattering angle $\theta$ in the laboratory frame versus the laboratory kinetic energy for the four reactions $K^{-} p \rightarrow \Sigma^{0} \pi^{0}, K^{-} p \rightarrow \Sigma^{0} \gamma, K^{-} p \rightarrow$ $n K_{S}^{0}$, and $K^{-} p \rightarrow n \overline{K^{0}} \pi^{0}$ at 600 and $740 \mathrm{MeV} / c$ incident beam momenta. 


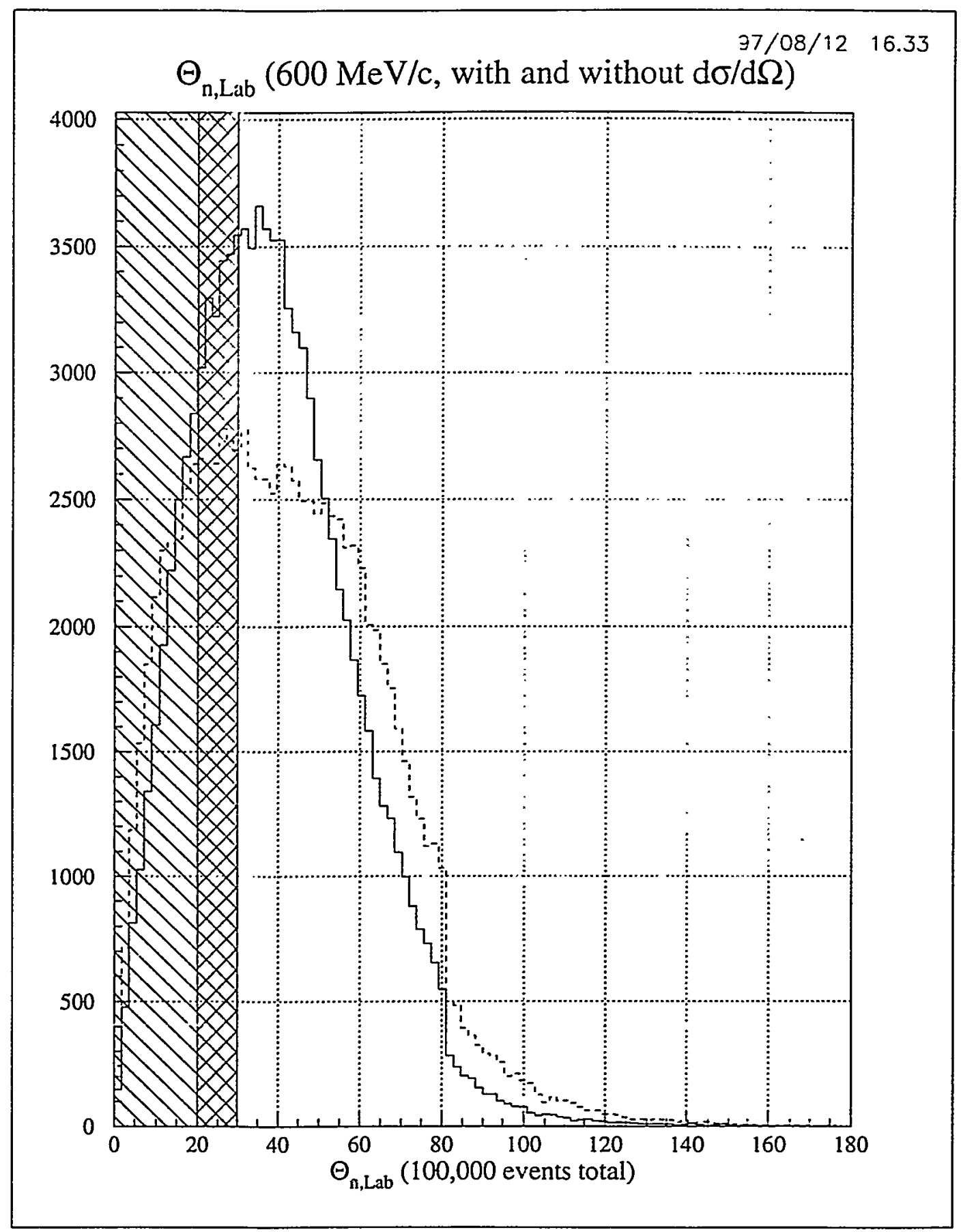

Figure 7: Plot of the number of events versus the laboratory scattering angle, $\theta_{n, L a b}$, for the sum of the six $\mathrm{K}^{-} p$ reactions described in the text at $600 \mathrm{MeV} / \mathrm{c}$ incident beam momentum. The solid curve assumes isotropic C.M. angular distributions, while the dashed curve includes information from measured differential cross sections. The three different angular regions are shown. 


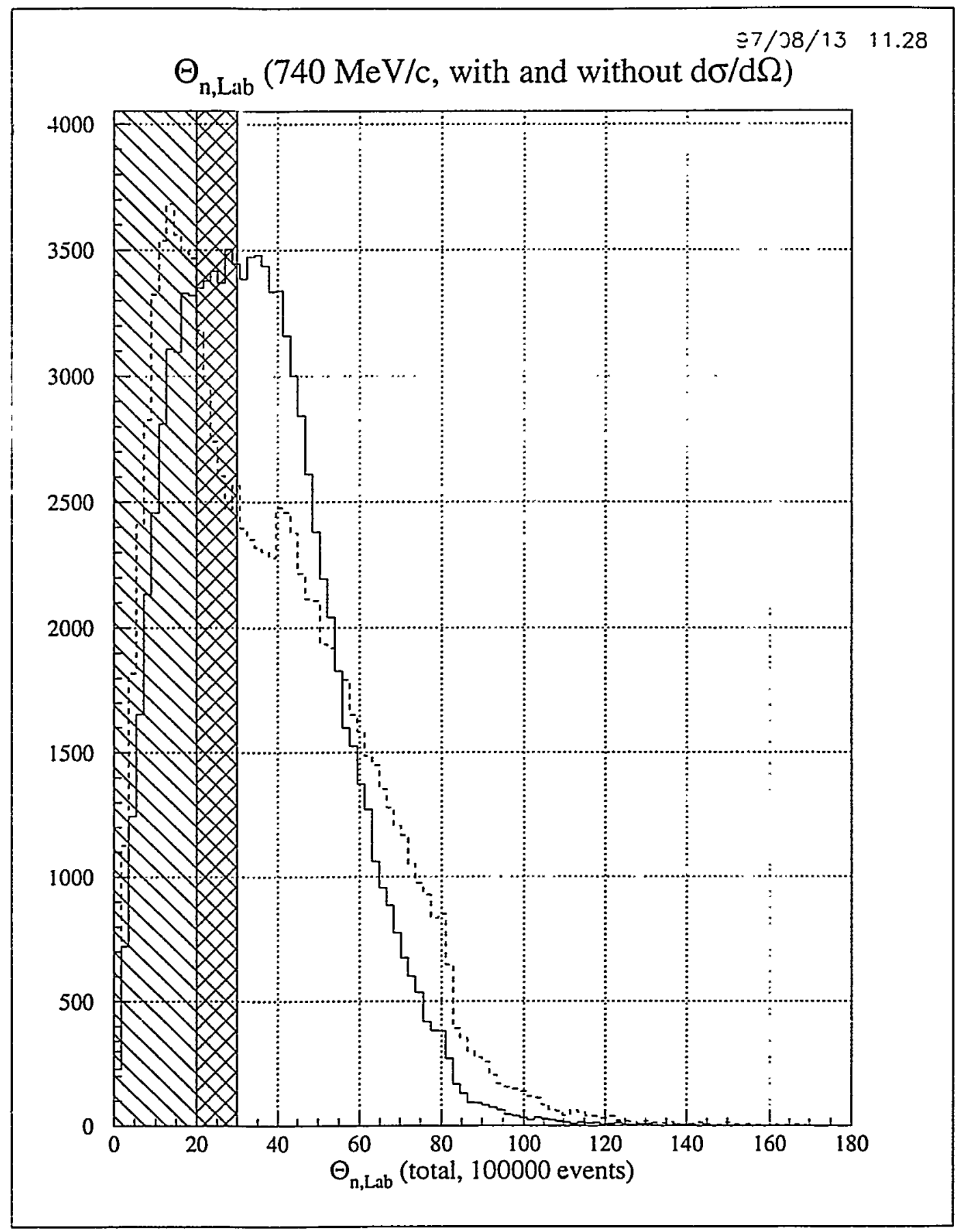

Figure 8: Plot of the number of events versus the laboratory scattering angle, $\theta_{n, L a b}$, for the sum of the six $\pi^{-} p$ reactions described in the text at $740 \mathrm{MeV} / \mathrm{c}$ incident beam momentum. The solid curve assumes isotropic C.M. angular distributions, while the dashed curve includes information from measured differential cross sections. The three different angular regions are shown. 


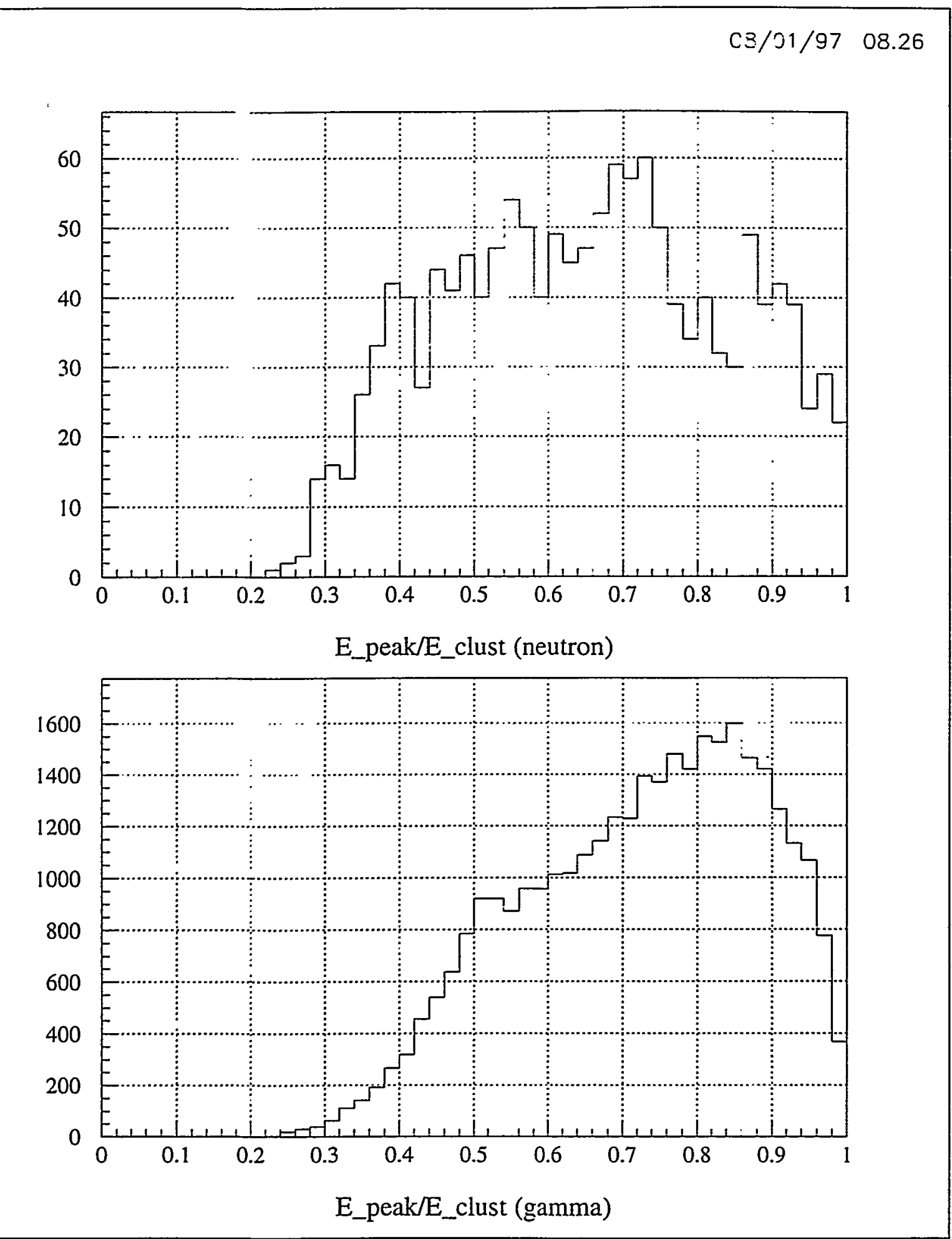

Figure 9: Histograms of the ratio $r_{1}$ from Eq. 2 for neutron and photon clusters from the $K^{-} p \rightarrow \Lambda^{0} \pi^{0}$ reaction. 


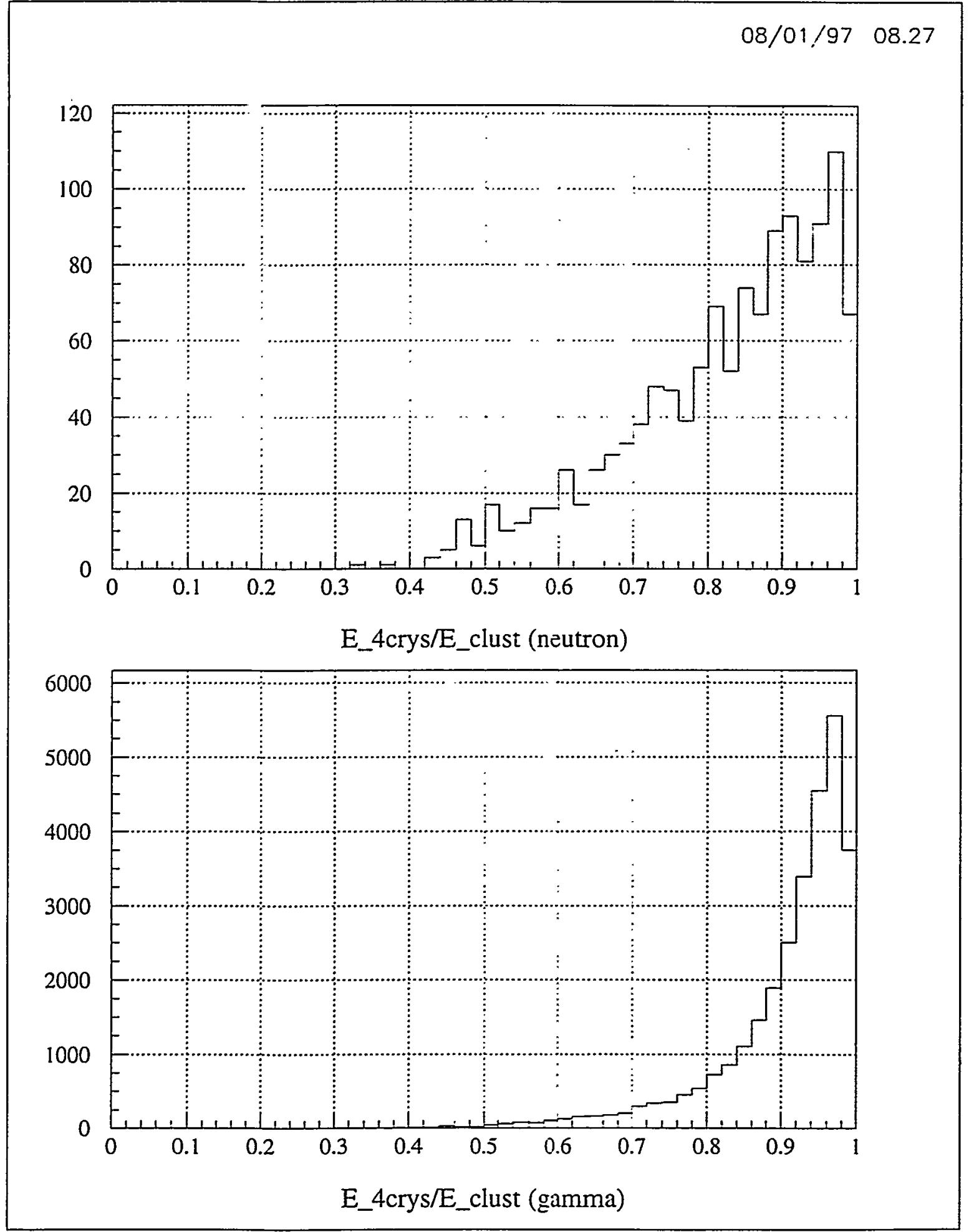

Figure 10: Histograms of the ratio $r_{4}$ from Eq. 3 for neutron and photon clusters from the $K^{-} p \rightarrow \Lambda^{0} \pi^{0}$ reaction. 


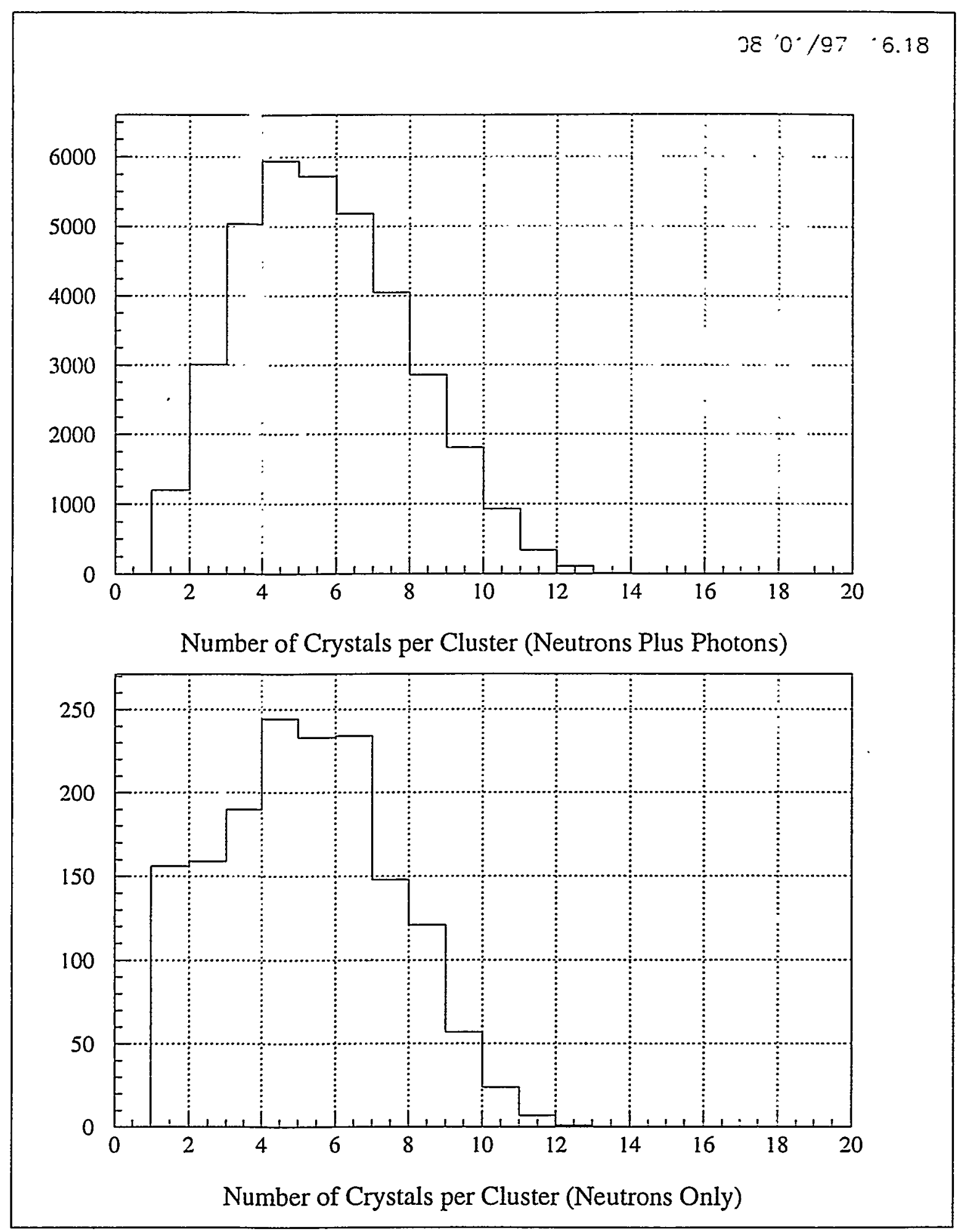

Figure 11: Histograms of the number of crystals per cluster and the number of crystals per neutron emitted in the $K^{-} p \rightarrow \Lambda^{0} \pi^{0}$ reaction. 


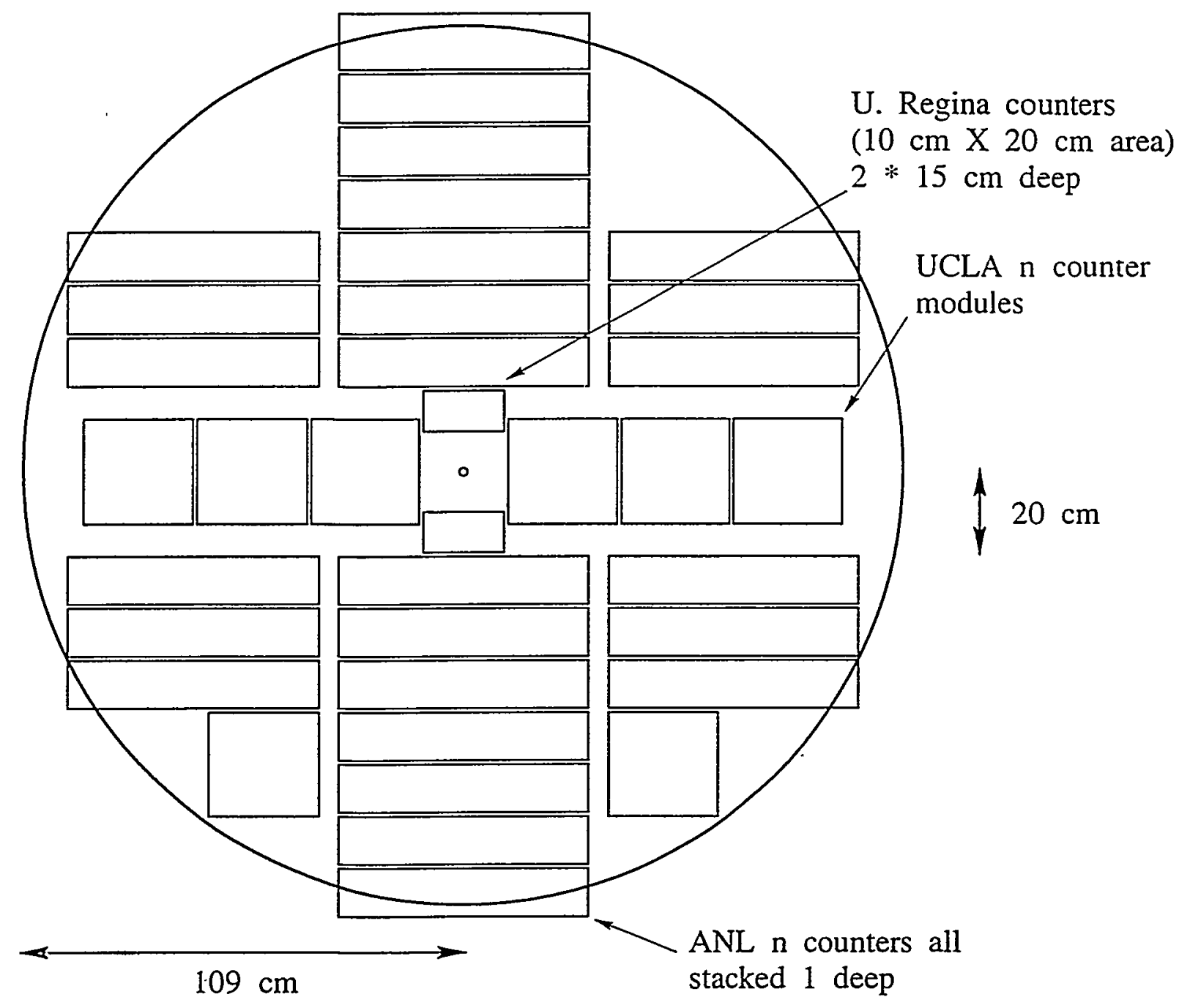

\section{CONFIGURATION A}

Figure 12: Diagram of the neutron counters in configuration A. The large circle defines the $20^{\circ}$ aperture of the Crystal Ball at $300 \mathrm{~cm}$, the small circle denotes the beam center, and the rectangles define the plastic scintillation neutron counters. 


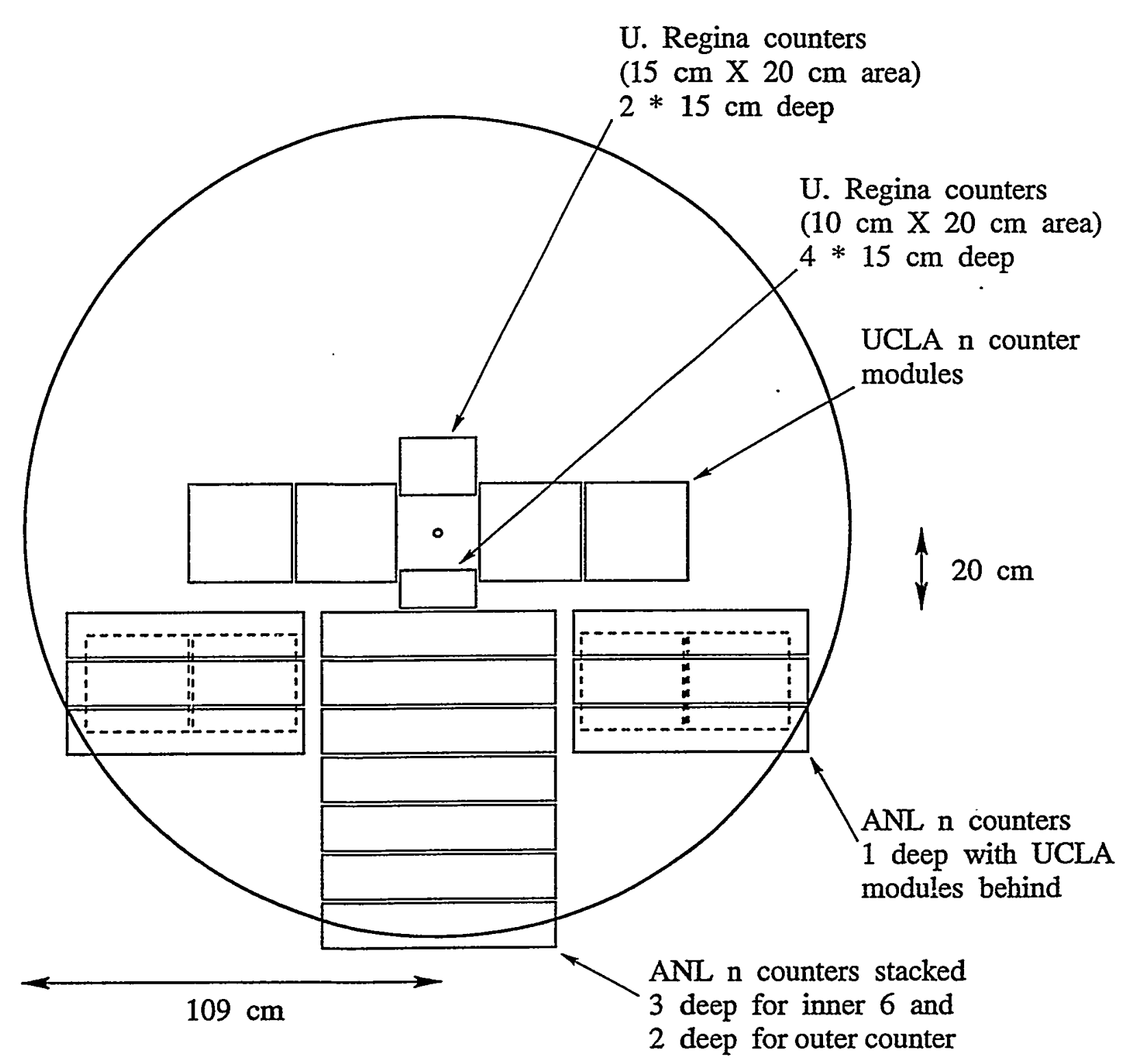

CONFIGURATION B

Figure 13: Diagram of the neutron counters in configuration B. 


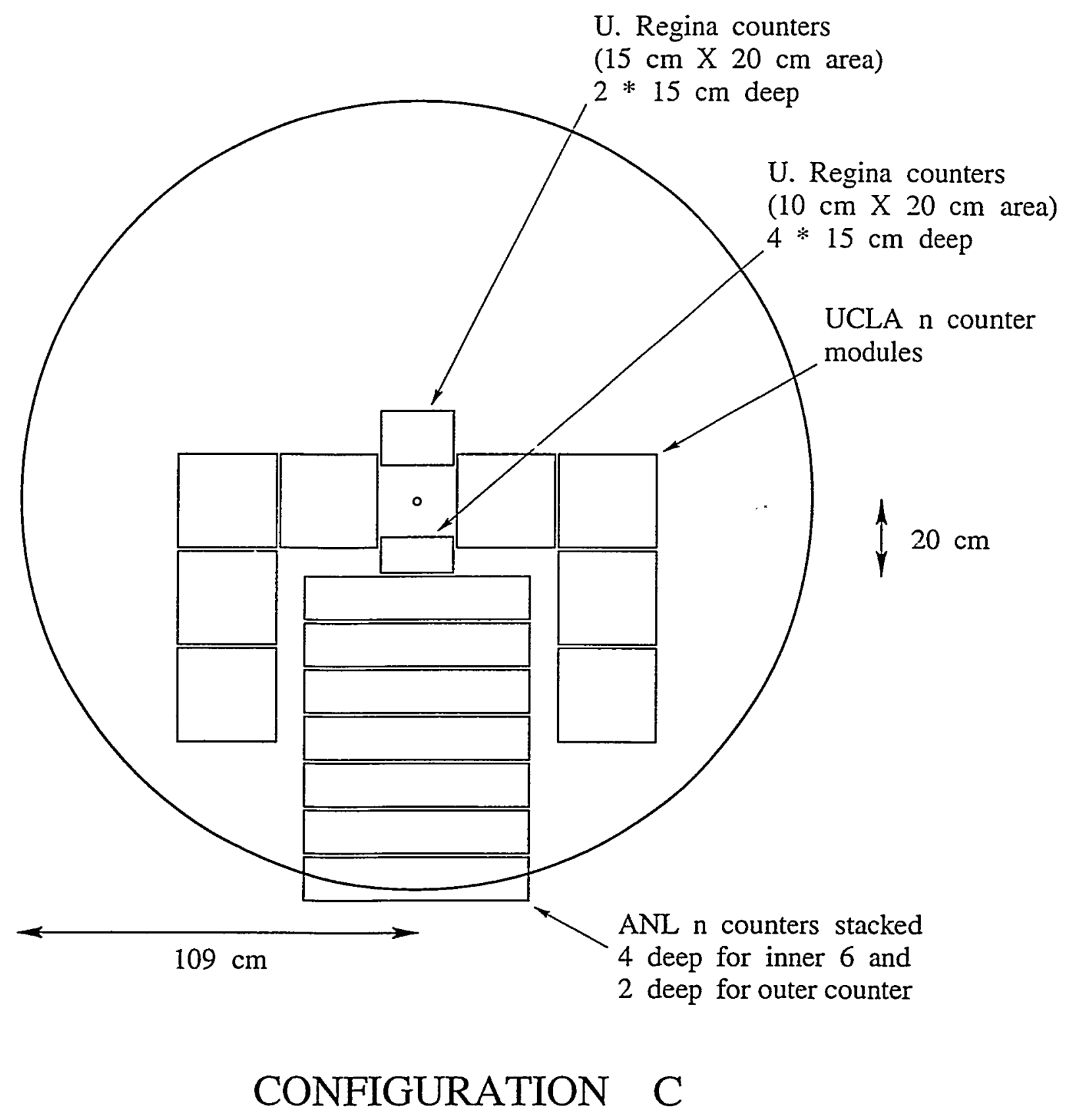

Figure 14: Diagram of the neutron counters in configuration C. 


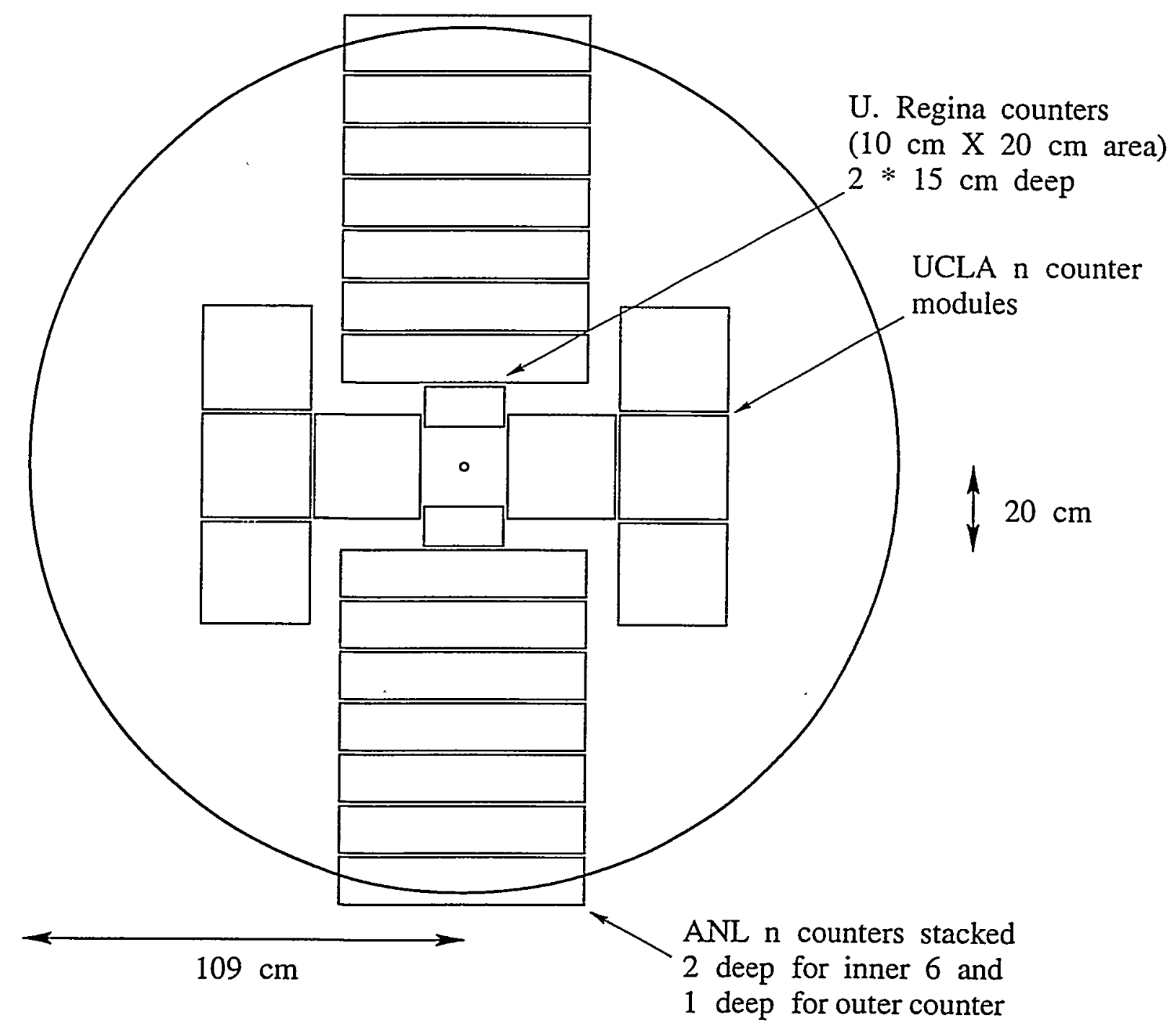

CONFIGURATION D

Figure 15: Diagram of the neutron counters in configuration D. 